\title{
Comprehensive analysis of data pertaining to the weak neutral current and the intermediate-vector-boson masses
}

\author{
Ugo Amaldi \\ CERN, Geneva, Switzerland \\ Albrecht Böhm \\ III. Physikalisches Institut A, Rheinisch West fälische Technische Hochschule, Aachen, West Germany \\ L. S. Durkin \\ Ohio State University, Columbus, Ohio 43210 \\ Paul Langacker and Alfred K. Mann \\ University of Pennsylvania, Philadelphia, Pennsylvania 19104 \\ William J. Marciano \\ Brookhaven National Laboratory, Upton, New York 11973 \\ Alberto Sirlin* \\ Rockefeller University, New York, New York 10021 \\ H. H. Williams \\ University of Pennsylvania, Philadelphia, Pennsylvania 19104 \\ (Received 14 May 1987)
}

\begin{abstract}
The results of a comprehensive analysis of existing data on the weak neutral current and the $W$ and $Z$ masses are presented. The principal results are the following. (a) There is no evidence for any deviation from the standard model. (b) A global fit to all data yields $\sin ^{2} \theta_{W} \equiv 1-M_{W}{ }^{2} / M_{Z}{ }^{2}=0.230 \pm 0.0048$, where this error and all others given here include full statistical, systematic, and theoretical uncertainties (computed assuming three fermion families, $m_{t} \leq 100 \mathrm{GeV}$, and $\left.\boldsymbol{M}_{H} \leq 1 \mathrm{TeV}\right)$. (c) Allowing $\rho \equiv \boldsymbol{M}_{W}{ }^{2} /\left(\boldsymbol{M}_{Z}{ }^{2} \cos ^{2} \theta_{W}\right)$ as well as $\sin ^{2} \theta_{W}$ to vary one obtains $\sin ^{2} \theta_{W}=0.229 \pm 0.0064$ and $\rho=0.998 \pm 0.0086$. This implies $90 \%$-confidence-level (C.L.) upper limits of 0.047 and 0.081 for the vacuum expectation values (relative to those of Higgs doublets) for Higgs triplets with weak hypercharge of 0 and \pm 1 , respectively. (d) The parameter $\delta_{W} \equiv \Delta r-\Delta s^{2}(1-\Delta r) / \sin ^{2} \theta^{0}$, which is a measure of the radiative corrections relating deepinelastic neutrino scattering, the $W$ and $Z$ masses, and muon decay, is determined to be $0.112 \pm 0.037$. This is consistent with the value $\delta_{W}=0.106$ expected for $m_{t}=45 \mathrm{GeV}$ and $M_{H}=100 \mathrm{GeV}$ and establishes the existence of radiative corrections at the $3 \sigma$ level. (e) The radiative corrections are sensitive to isospin breaking associated with a large $m_{t}$. Assuming no deviation from the standard model, consistency of the various reactions requires $m_{t}<180 \mathrm{GeV}$ at $90 \%$ C.L. for $M_{H} \leq 100 \mathrm{GeV}$, with a slightly weaker limit for larger $M_{H}$. Similar results hold for the mass splittings between fourth-generation quarks or leptons. (f) Most of the parameters in modelindependent fits to $v q$, $v e, e q$, and $e^{+} e^{-}$processes are now determined uniquely and precisely. (g) Limits are given on the masses and mixing angles of additional $Z$ bosons expected in popular models. For theoretically expected coupling constants one finds that the neutral-current constraints are usually more stringent than the direct-production limits from the CERN S $\bar{p} p$ S collider, but nevertheless masses as low as $120-300 \mathrm{GeV}$ are typically allowed. (h) The implications of these results for grand unification are discussed. $\sin ^{2} \theta_{W}$ is $\geq 2.5$ standard deviations above the prediction of minimal SU(5) and similar models for all $m_{t}$. It is closer to the prediction of simple supersymmetric grand unified theories but is still somewhat low. (i) The dominant theoretical uncertainty (the charm-quark threshold in deep-inelastic charged-current scattering) is considered in some detail.
\end{abstract}

\section{INTRODUCTION}

The weak neutral current and the $W$ and $Z$ masses have been major quantitative tests of the standard $\mathrm{SU}(2) \times \mathrm{U}(1)$ electroweak model, ${ }^{1}$ the predictions of which have been verified for a variety of probes and reactions at momentum scales ranging roughly from 1 $\mathrm{MeV}$ to $100 \mathrm{GeV}$.

Recently, many new high-precision experiments in deep-inelastic scattering from isoscalar and nonisoscalar 
targets, $v p$ elastic scattering, $v e$ scattering, atomic parity violation, $e^{+} e^{-}$annihilation, and the $W$ and $Z$ masses have been completed. As we await a new generation of even more precise experiments, mainly at $e^{+} e^{-}, e p$, and hadron colliders, it is worthwhile to make a systematic and critical study of existing results. ${ }^{2-12}$ Our motivations for this study are to test the standard model at the level of radiative corrections, to extract as accurate a value of $\sin ^{2} \theta_{W}$ as possible for comparison with grand unified theories, supersymmetry, etc., and to search for indications of (or set limits on) such new physics as additional $Z$ bosons, heavy fermions, or nonstandard Higgs representations.

We have attempted to incorporate all published data on $v$-hadron, $v e, e N, \mu N$, and $e^{+} e^{-}$reactions, atomic parity violation, and the $W$ and $Z$ masses, with the exception of those for which, in our opinion, either experimental uncertainties or difficulties in the theoretical interpretation preclude useful or reliable quantitative constraints. The data are summarized in Tables I-XI along with the predictions of the standard model for $\sin ^{2} \theta_{W}=0.230$. We have also tried to use the best available theoretical expressions, to make realistic estimates of theoretical uncertainties, and to ensure that similar experiments are treated as uniformly as possible. Electroweak radiative corrections are included ${ }^{13,14}$ except where their effects are negligible compared to the experimental uncertainties. Statistical, systematic, and theoretical $^{15}$ uncertainties are generally combined in quadrature, except in a few cases for which it is useful to display the theoretical uncertainties separately, and all available error correlations are included. Fits to the data were carried out using the computer program MINUIT (Ref. 16). In this paper we present the major results. Fuller details of the experiments, theoretical expressions, radiative corrections, and error analysis will be presented elsewhere. ${ }^{17}$

\section{NOTATION AND THEORETICAL EXPRESSIONS}

The effective Lagrangian for low-energy neutralcurrent interactions between massless left-handed neutrinos and quarks is

$$
-L^{v H}=\frac{G_{F}}{\sqrt{2}} \bar{v} \gamma^{\mu}\left(1+\gamma_{5}\right) v J_{\mu}^{H}
$$

where

$$
\begin{aligned}
J_{\mu}^{H} & =\sum_{i}\left[\epsilon_{L}(i) \bar{q}_{i} \gamma_{\mu}\left(1+\gamma_{5}\right) q_{i}+\epsilon_{R}(i) \bar{q}_{i} \gamma_{\mu}\left(1-\gamma_{5}\right) q_{i}\right] \\
& =\sum_{i} \bar{q}_{i} \gamma_{\mu}\left(g_{V}^{i}+g_{A}^{i} \gamma_{5}\right) q_{i}
\end{aligned}
$$

where $g_{V, A}^{i} \equiv \epsilon_{L}(i) \pm \epsilon_{R}(i)$. In (1) we have assumed identical couplings for all neutrino flavors and neglected possible $S, P, T$ interactions. We will assume family independence of the quark couplings: $\epsilon_{L, R}(u)=\epsilon_{L, R}(c)$, etc. In the standard model,

$$
\begin{aligned}
& \epsilon_{L}(u)=\rho_{v N}^{\mathrm{NC}}\left(\frac{1}{2}-\frac{2}{3} \kappa_{v N} \sin ^{2} \theta_{W}+\lambda_{u_{L}}\right), \\
& \epsilon_{L}(d)=\rho_{v N}^{\mathrm{NC}}\left(-\frac{1}{2}+\frac{1}{3} \kappa_{v N} \sin ^{2} \theta_{W}+\lambda_{d_{L}}\right), \\
& \epsilon_{R}(u)=\rho_{v N}^{\mathrm{NC}}\left(-\frac{2}{3} \kappa_{v N} \sin ^{2} \theta_{W}+\lambda_{u_{R}}\right), \\
& \epsilon_{R}(d)=\rho_{v N}^{\mathrm{NC}}\left(\frac{1}{3} \kappa_{v N} \sin ^{2} \theta_{W}+\lambda_{d_{R}}\right),
\end{aligned}
$$

where $^{14} \rho_{v N}^{\mathrm{NC}}=1.00074, \quad \kappa_{v N}=0.9902, \quad \lambda_{u_{L}}=-0.0031$, $\lambda_{d_{L}}=-0.0026$, and $\lambda_{u_{R}}=\frac{1}{2} \lambda_{d_{R}}=3.5 \times 10^{-5}$ are radiative-correction parameters. (The central values of all radiative corrections are calculated for $\sin ^{2} \theta_{W}=0.23$, $m_{t}=45 \mathrm{GeV}$, and $M_{H}=100 \mathrm{GeV}$, where $m_{t}$ and $M_{H}$ are the masses of the top quark and Higgs boson, respectively.)

Similarly, the interaction between $\left.{ }^{v}\right)_{\mu}$ and electrons is described by

$$
-L^{v e}=\frac{G_{F}}{\sqrt{2}} \bar{v}_{\mu} \gamma^{\mu}\left(1+\gamma_{5}\right) v_{\mu} J_{\mu}^{e}
$$

where

$$
J_{\mu}^{e}=\bar{e} \gamma_{\mu}\left(g_{V}^{e}+g_{A}^{e} \gamma_{5}\right) e
$$

(for $\left.{ }^{v}\right)_{e} e$ the charged-current contribution must be included). In the standard model

$$
g_{V}^{e}=\rho_{v e}\left(-\frac{1}{2}+2 \kappa_{v e} \sin ^{2} \theta_{W}\right), \quad g_{A}^{e}=\rho_{v e}\left(-\frac{1}{2}\right),
$$

where $\kappa_{v e}=0.9897$ and $\mu_{v e}=1.0054$. The most precise measurements involve the ratio $\sigma_{v_{\mu} e} / \sigma_{\bar{v}_{\mu} e}$, in which $\rho_{v e}$ cancels.

The effective parity-violating interaction between electrons and quarks is

$$
-L^{e H}=\frac{G_{F}}{\sqrt{2}} \sum_{i}\left(C_{1 i} \bar{e} \gamma_{\mu} \gamma_{5} e \bar{q}_{i} \gamma^{\mu} q_{i}+C_{2 i} \bar{e} \gamma_{\mu} e \bar{q}_{i} \gamma^{\mu} \gamma_{5} q_{i}\right) \text {. }
$$

In $\mathrm{SU}(2) \times \mathbf{U}(1)$ one has

$$
\begin{aligned}
& C_{1 u}=\rho_{e q}^{\prime}\left(-\frac{1}{2}+\frac{4}{3} \kappa_{e q}^{\prime} \sin ^{2} \theta_{W}\right), \\
& C_{1 d}=\rho_{e q}^{\prime}\left(\frac{1}{2}-\frac{2}{3} \kappa_{e q}^{\prime} \sin ^{2} \theta_{W}\right), \\
& C_{2 u}=-C_{2 d}=\rho_{e q}\left(-\frac{1}{2}+2 \kappa_{e q} \sin ^{2} \theta_{W}\right),
\end{aligned}
$$

where negligibly small constants have been neglected. For atomic parity violation, $\rho_{e q}^{\prime}=0.9793$ and $\kappa_{e q}^{\prime}=0.9948$. For the SLAC polarized-electron experiment $\rho_{e q}^{\prime}=0.970, \kappa_{e q}^{\prime}=0.993, \rho_{e q}=0.993$, and $\kappa_{e q}=1.03$ after incorporating additional QED corrections.

The total cross section (relative to pure QED) and forward-backward asymmetry for $e^{+} e^{-} \rightarrow l l, l=\mu$ or $\tau$, are given in the standard model by

$$
R_{l l}=F_{1}, \quad A_{l l}=3 F_{2} / 4 F_{1}
$$

where

$$
\begin{aligned}
& F_{1}=1-2 \chi h_{V V} \cos \delta_{R}+\left(h_{V V}+h_{A A}\right)^{2} \chi^{2}, \\
& F_{2}=-2 \chi h_{A A} \cos \delta_{R}+4 \chi^{2} h_{V V} h_{A A} .
\end{aligned}
$$




$$
\begin{aligned}
& \text { In (10), } \\
& h_{V V}=\left(g_{V}^{0}\right)^{2}=\left(-\frac{1}{2}+2 \sin ^{2} \theta_{W}\right)^{2}, \\
& h_{A A}=\left(g_{A}^{0}\right)^{2}=\left(-\frac{1}{2}\right)^{2} \text {, } \\
& \tan \delta_{R}=\frac{M_{Z} \Gamma_{Z}}{M_{Z}{ }^{2}-s}, \\
& \chi=\frac{G_{F}\left(1-\Delta_{0}\right)}{2 \sqrt{2} \pi \alpha} \frac{s M_{Z}^{2}}{\left[\left(m_{Z}^{2}-s\right)^{2}+m_{Z}^{2} \Gamma_{Z}^{2}\right]^{1 / 2}},
\end{aligned}
$$

where $\sqrt{s}$ is the c.m. energy. $\Delta_{0}=0.0713$ represents approximately the remaining radiative corrections ${ }^{18}$ in current experiments after pure QED corrections have been subtracted from the data. At the energies of the SLAC and DESY $e^{+} e^{-}$storage rings PEP and PETRA, $A_{l l}$ is insensitive to $\sin ^{2} \theta_{W}$. The major $\sin ^{2} \theta_{W}$ constraint is from the weak-electromagnetic interference $(\chi)$ term in $R_{l l}$, which would be important for $\sin ^{2} \theta_{W}$ much different from $\frac{1}{4}$. In fact, for $\sin ^{2} \theta_{W}$ near 0.23 the pure weak $\left(\chi^{2}\right)$ term in $F_{1}$ is numerically larger than the weak-electromagnetic interference term.

Finally, the $W$ and $Z$ masses are predicted to be

$$
M_{W}=\frac{A_{0}}{\sin \theta_{W}(1-\Delta r)^{1 / 2}}, \quad M_{Z}=\frac{M_{W}}{\cos \theta_{W}},
$$

where $A_{0}=\left(\pi \alpha / \sqrt{2} G_{F}\right)^{1 / 2}=37.281 \mathrm{GeV}$.

The radiative-correction parameter $\Delta r$ is predicted to be $0.0713 \pm 0.0013$ for $m_{t}=45 \mathrm{GeV}, M_{H}=100 \mathrm{GeV}$. As is apparent from (13) the renormalized weak angle is defined ${ }^{14}$ as $\sin ^{2} \theta_{W} \equiv 1-M_{W}{ }^{2} / M_{Z}{ }^{2}$ in the standard model.

\section{DATA}

The results of 13 high-energy experiments ${ }^{19-32}$ on deep-inelastic $\left.v_{\mu}\right)_{\mu}$ scattering from approximately isoscalar targets are listed in Table I. The analysis is dominated by four recent high-precision experiments at CERN (CDHS, CHARM) and Fermilab (CCFRR, FMM), but the stability of the central values of $R_{v}$ and $R_{\bar{v}}$ over many years is also displayed in Table $I$.

The CDHS and CHARM groups have avoided a number of theoretical uncertainties by using a formalism ${ }^{33}$ in which many details of the hadronic structure functions are absorbed into the empirical value of $r \equiv \sigma_{\bar{v} N}^{\mathrm{CC}} / \sigma_{v N}^{\mathrm{CC}}$. They find $\sin ^{2} \theta_{W}=0.225 \pm 0.007$ (CDHS) and $0.236 \pm 0.007$ (CHARM) for $m_{c}=1.5 \pm 0.3 \mathrm{GeV}$ (Ref. 34), where we have combined statistical and systematic uncertainties in quadrature. This formalism is easily generalized to other electroweak models (which are needed for our analysis), but unfortunately the necessary $r$ values are not available for many of the deep-inelastic experiments. We therefore use instead a QCD-improved parton model for the deep-inelastic structure functions. This is justified by the conclusion of Ref. 33 and other analyses $^{35}$ that the higher-twist corrections are very small.

For all deep-inelastic experiments except CCFRR and FMM we have derived "model-independent" expressions of the form $R_{v}=\sum_{i}\left[a_{i} \epsilon_{L}(i)^{2}+b_{i} \epsilon_{R}(i)^{2}\right]$ (and similarly for $R_{\bar{v}}$ ). These are computed by folding the $Q^{2}$ dependent quark distribution functions with the appropriate neutrino spectra and cuts for each reaction and applying corrections for nonisoscalar targets where necessary. The shapes of the valence and sea-quark distributions and $\bar{U}+\bar{D}, S$, and $C$, which are the first moments of $\bar{u}(x)+\bar{d}(x), s(x)$, and $c(x)$, respectively, are specified at $Q_{0}{ }^{2}=5 \mathrm{GeV}^{2}$ to agree with charged-current data, and the distributions evolve in $Q^{2}$ to reproduce the correct QCD $Q^{2}$ dependence of the first two moments (similar in spirit to the Buras-Gaemers distributions ${ }^{36}$ ). In addition, a correction is made for the longitudinal structure function $R_{L}=\sigma_{L} / \sigma_{T}$, full electroweak radiative corrections are applied to the charged-current cross section, ${ }^{14}$ and hadron leg photonic corrections are applied to the distribution functions in both $\sigma^{\mathrm{NC}}$ and $\sigma^{\mathrm{CC}}$. Other (standard-model-dependent) radiative corrections are incorporated into the expressions for the $\epsilon$ 's in (3). The charm-quark threshold, which is mainly important in the charged-current denominator in $R_{v}$, is treated assuming slow rescaling ${ }^{37}$ with an effective charm-quark mass of $m_{c}=1.5 \pm 0.3 \mathrm{GeV}$.

Theoretical uncertainties in the determination of $\sin ^{2} \theta_{W}$ from deep-inelastic scattering are estimated by varying all structure-function parameters over reasonable ranges and by using independent estimates of the effects of higher twist. ${ }^{33,35}$ Most theoretical uncertainties are very small because all that enters the neutralcurrent analysis are the ratios of the (appropriately weighted) first moments of the various quark distribution functions. For three fermion families the major uncertainty turns out to be the charm-quark threshold. This uncertainty, which is represented by the range $1.5 \pm 0.3$ $\mathrm{GeV}$ allowed for $m_{c}$, is propagated through all of the other fits as well. There is also some apparent sensitivity to the shapes of the quark $x$ distributions [especially $s(x)$ ], but this is actually due almost entirely to the $c$ threshold and is not independent of $m_{c}$. The $c$ threshold, slow rescaling, and their implications for dimuons are further discussed in the Appendix.

As a consistency check, the central values for $\sin ^{2} \theta_{W}$ that we obtain from the new CDHS and CHARM results are 0.2260 and 0.2377 , respectively, in excellent agreement with the central values 0.225 and 0.236 obtained by CDHS and CHARM in their own analyses. The analyses are not totally independent because we have adjusted $(\bar{U}+\bar{D}) /(U+D)$ at $Q_{0}{ }^{2}$ to agree with the value $^{38} r=0.39 \pm 0.01$ measured by CDHS, and because we have used similar values for $m_{c}, \bar{S} / \bar{U}, C / S$, and $D_{V} / U_{V}\left(D_{V}\right.$ and $U_{V}$ are the first moments of the valence $d$ and $u$ quarks, respectively). However, our models for the $x$ and $Q^{2}$ dependence of the structure functions and our implementation of the radiative corrections are completely independent. [The agreement is actually better than it appears because we have incorporated a reevaluation of the $\alpha\left(Q^{2}\right)$-dependent effects in the radiative corrections that systematically increases $\sin ^{2} \theta_{W}$ by $\sim 0.001$.] We therefore find the agreement to be significant.

For CCFRR and FMM we have used values of $g_{L, R}{ }^{2}=\epsilon_{L, R}(u)^{2}+\epsilon_{L, R}(d)^{2}$ extracted from the groups' 
TABLE I. Results for deep-inelastic scattering from approximately isoscalar targets. $R_{v} \equiv \sigma_{v N}^{\mathrm{NC}} / \sigma_{v N}^{\mathrm{CC}}$ and $R_{\bar{v}} \equiv \sigma_{\bar{v} N}^{\mathrm{NC}} / \sigma_{\bar{v} N}^{\mathrm{CC}}$ where $\mathrm{NC}$ and CC represent neutral current and charged current, respectively, while $g_{i}{ }^{2} \equiv \epsilon_{i}(u)^{2}+\epsilon_{i}(d)^{2}$ for $i=L$ or $R$. The numbers in parentheses below the experimental values are the standard-model predictions for $\sin ^{2} \theta_{W}=0.230$.

\begin{tabular}{|c|c|c|c|}
\hline Group & Experimental & value & Reference \\
\hline $\begin{array}{l}\text { CDHS (CERN) } \\
\text { (iron) }\end{array}$ & $\begin{array}{l}R_{v}=0.3072 \pm 0.0032 \\
(0.3035)\end{array}$ & & 19 \\
\hline $\begin{array}{l}\text { CHARM (CERN) } \\
\quad \text { (marble-isoscalar corrected) }\end{array}$ & $\begin{array}{l}R_{v}=0.3093 \pm 0.0031 \\
(0.3127)\end{array}$ & & 20 \\
\hline $\begin{array}{l}\text { CCFRR (Fermilab) } \\
\text { (iron) }\end{array}$ & $\begin{array}{l}g_{L}^{2}=0.292 \pm 0.009 \\
(0.301)\end{array}$ & $\begin{array}{l}g_{R}^{2}=0.030 \pm 0.009 \\
(0.029)\end{array}$ & 21 \\
\hline $\begin{array}{l}\text { FMM (Fermilab) } \\
\text { (sand-steel) }\end{array}$ & $\begin{array}{l}g_{L}^{2}=0.282 \pm 0.014 \\
(0.301)\end{array}$ & $\begin{array}{l}g_{R}^{2}=0.044 \pm 0.014 \\
(0.029)\end{array}$ & 22 \\
\hline $\begin{array}{l}\text { CDHS (CERN) } \\
\text { (iron) }\end{array}$ & $\begin{array}{l}R_{v}=0.301 \pm 0.007 \\
(0.303)\end{array}$ & $\begin{array}{l}R_{\bar{v}}=0.363 \pm 0.015 \\
(0.375)\end{array}$ & 23 \\
\hline $\begin{array}{l}\text { SKAT (Serpukhov) } \\
\text { (freon) }\end{array}$ & $\begin{array}{l}R_{v}=0.33 \pm 0.02 \\
(0.32)\end{array}$ & $\begin{array}{l}R_{\bar{v}}=0.44 \pm 0.11 \\
(0.40)\end{array}$ & 24 \\
\hline $\begin{array}{l}\text { FIIM (Fermilab } 15 \mathrm{ft}) \\
\text { (neon-hydrogen) }\end{array}$ & & $\begin{array}{l}R_{\bar{v}}=0.406 \pm 0.028 \\
(0.367)\end{array}$ & 25 \\
\hline $\begin{array}{l}\text { ABCDILOS (CERN-BEBC) } \\
\text { (neon-hydrogen) }\end{array}$ & $\begin{array}{l}R_{v}=0.345 \pm 0.018 \\
(0.313)\end{array}$ & $\begin{array}{l}R_{\bar{v}}=0.364 \pm 0.030 \\
(0.364)\end{array}$ & 26 \\
\hline $\begin{array}{l}\text { ABBPPST (CERN-BEBC) } \\
\text { (deuterium) }\end{array}$ & $\begin{array}{l}R_{v}=0.33 \pm 0.03 \\
(0.31)\end{array}$ & $\begin{array}{l}R_{\bar{v}}=0.35 \pm 0.05 \\
(0.37)\end{array}$ & 27 \\
\hline $\begin{array}{l}\text { Fermilab } 15 \mathrm{ft} \\
\text { (neon-hydrogen) }\end{array}$ & $\begin{array}{l}R_{v}=0.32 \pm 0.03 \\
(0.33)\end{array}$ & & 28 \\
\hline $\begin{array}{l}\text { CHARM (CERN) } \\
\text { (marble) }\end{array}$ & $\begin{array}{l}R_{v}=0.320 \pm 0.010 \\
(0.314)\end{array}$ & $\begin{array}{l}R_{\bar{v}}=0.377 \pm 0.020 \\
(0.362)\end{array}$ & 29 \\
\hline $\begin{array}{l}\text { CITF (Fermilab) } \\
\text { (iron) }\end{array}$ & $\begin{array}{l}R_{v}=0.28 \pm 0.03 \\
(0.30)\end{array}$ & $\begin{array}{l}R_{\bar{v}}=0.35 \pm 0.11 \\
(0.39)\end{array}$ & 30 \\
\hline $\begin{array}{l}\text { HPWF (Fermilab) } \\
\qquad\left(\mathrm{CH}_{2}\right)\end{array}$ & $\begin{array}{l}R_{v}=0.30 \pm 0.04 \\
(0.33)\end{array}$ & $\begin{array}{l}R_{\bar{v}}=0.33 \pm 0.09 \\
(0.35)\end{array}$ & 31 \\
\hline
\end{tabular}

published data. ${ }^{39}$ We have checked that their assumptions concerning quark distributions, radiative corrections, and the $c$ threshold are consistent with our own.

In addition, we have incorporated the results of nine deep-inelastic scattering experiments ${ }^{40-48}$ on nonisoscalar targets (Table II), which provide information on the isospin structure of the neutral current. We have applied the same type of analysis to these reactions as to the isoscalar-target experiments. Of particular importance is the use of $D_{V} / U_{V}=0.39 \pm 0.06$ at $Q_{0}{ }^{2}$, as determined in recent charged-current experiments. ${ }^{49}$ The naive ansatz $D_{V} / U_{V}=0.5$, used in earlier studies ${ }^{5,9}$ and by most of the experimental groups, yields considerably different results. (In particular the value of $\sin ^{2} \theta_{W}$ determined from the data assuming $D_{V} / U_{V}=0.5$ is lowered by $\simeq 0.05$ than the value obtained for $D_{V} / U_{V}=0.39$. ) We have propagated the uncertainty in $D_{V} / U_{V}$ through all fits.

Other neutrino-hadron data ${ }^{50-54}$ are displayed in Table III. For the recent high-precision (BNL E734) $\left(v_{\mu} p \rightarrow v_{\mu} p\right.$ experiment ${ }^{50,55,56}$ we use the worldaverage $^{57} M_{A}=1.032 \pm 0.036 \mathrm{GeV}$ for the axial-vector form-factor mass (propagating the uncertainty through all fits), and $\epsilon=0.125 \pm 0.125$ to represent the contribution of $c$ and $s$ quarks to the axial-vector matrix element. $^{58}$

We include the four recent measurements of coherent pion production $v N \rightarrow v \pi^{0} N$. The theoretical interpretation $^{59}$ of this reaction depends on a fairly reliable application of PCAC (partial conservation of axial-vector current) and a model of $\pi^{0}$-nucleus scattering that is verified to $15-20 \%$ in the cross section of the corresponding charged-current process. We have added a $10 \%$ theoretical uncertainty (in amplitude) to the \pm 0.12 experimental uncertainty quoted by AP. The CHARM and CB groups have already included theoretical uncertainties in their quoted errors, and the SKAT experiment avoids most theoretical uncertainties by using isospin arguments for the measured ratio of the neutraland charged-current coherent pion-production cross sections.

Data on inclusive and exclusive incoherent pion production $^{60}$ and on the reaction ${ }^{61} \bar{v}_{e} \mathrm{D} \rightarrow \bar{v}_{e} n p$ are in qualitative agreement with the standard model, but are not used here because of uncertainties in the theoretical matrix elements. These reactions were useful in earlier studies for determining the isospin structure of the $v q$ couplings (and especially in excluding the dominantly isoscalar solution), but their role is now superseded by the more easily interpreted reactions in Tables II and III.

The experimental results ${ }^{62-70}$ for $v e$ scattering are listed in Table IV. There are two recent high-precision experiments (CHARM and E734) on both $v_{\mu} e$ and $\bar{v}_{\mu} e$ 
TABLE II. Deep-inelastic scattering from nonisoscalar targets. $R_{v}^{p} \equiv \sigma_{v p}^{\mathrm{NC}} / \sigma_{v p}^{\mathrm{CC}}, R_{v}^{n} \equiv \sigma_{v n}^{\mathrm{NC}} / \sigma_{v n}^{\mathrm{CC}}$, and $R_{\bar{v}}^{p} \equiv \sigma_{\bar{v} p}^{\mathrm{NC}} / \sigma_{\bar{v} p}^{\mathrm{CC}}$, while $R_{v}^{n / p} \equiv \sigma_{v n}^{\mathrm{NC}} / \sigma_{v p}^{\mathrm{NC}}$ and $R_{\bar{v}}^{n / p} \equiv \sigma_{\bar{v} n}^{\mathrm{NC}} / \sigma_{\bar{v} p}^{\mathrm{NC}} . \quad r_{v}$ is defined as $\left(\sigma_{v p}^{\mathrm{NC}}-\sigma_{v n}^{\mathrm{NC}}\right) /$ $\left(\sigma_{v p}^{\mathrm{CC}}-\sigma_{v n}^{\mathrm{CC}}\right)$, with an analogous definition for $r_{\bar{v}}$. The numbers in parentheses are the standard-model predictions for $\sin ^{2} \theta_{W}=0.230$.

\begin{tabular}{|c|c|c|c|}
\hline Group & Experimental & value & Reference \\
\hline $\begin{array}{l}\text { BBCIMOU (CERN-BEBC) } \\
\text { (hydrogen) }\end{array}$ & $\begin{array}{l}R_{v}^{p}=0.384 \pm 0.028 \\
(0.403)\end{array}$ & $\begin{array}{l}R_{\frac{p}{v}}^{p}=0.338 \pm 0.021 \\
(0.333)\end{array}$ & 40 \\
\hline $\begin{array}{l}\text { BEBC-TST (CERN) } \\
\text { (hydrogen) }\end{array}$ & $\begin{array}{l}R_{v}^{p}=0.47 \pm 0.04 \\
(0.45)\end{array}$ & $\begin{array}{l}R_{\bar{v}}^{p}=0.33 \pm 0.04 \\
(0.28)\end{array}$ & 41 \\
\hline $\begin{array}{l}\text { SIMTT (Fermilab } 15 \mathrm{ft} \text { ) } \\
\text { (deuterium) }\end{array}$ & $\begin{array}{l}R_{v}^{p}=0.49 \pm 0.06 \\
(0.44) \\
R_{v}^{n}=0.22 \pm 0.03 \\
(0.23)\end{array}$ & & 42 \\
\hline $\begin{array}{l}\text { Fermilab } 15 \mathrm{ft} \\
\text { (hydrogen) }\end{array}$ & & $\begin{array}{l}R \frac{p}{v}=0.36 \pm 0.06 \\
(0.28)\end{array}$ & 43 \\
\hline $\begin{array}{l}\text { ABCMO (CERN-BEBC) } \\
\text { (hydrogen) }\end{array}$ & $\begin{array}{l}R_{v}^{p}=0.51 \pm 0.04 \\
(0.52)\end{array}$ & & 44 \\
\hline $\begin{array}{l}\text { Fermilab } 15 \mathrm{ft} \\
\quad \text { (hydrogen) }\end{array}$ & $\begin{array}{l}R_{v}^{p}=0.48 \pm 0.17 \\
(0.46)\end{array}$ & & 45 \\
\hline $\begin{array}{l}\text { FIIM (Fermilab } 15 \mathrm{ft} \text { ) } \\
\text { (neon-hydrogen) }\end{array}$ & & $\begin{array}{l}R_{\bar{v}}^{n / p}=0.88 \pm 0.17 \\
(1.03)\end{array}$ & 46 \\
\hline $\begin{array}{l}\text { Fermilab } 15 \mathrm{ft} \\
\text { (neon-hydrogen) }\end{array}$ & $\begin{array}{l}R_{v}^{n / p}=1.08 \pm 0.19 \\
(1.13)\end{array}$ & & 47 \\
\hline $\begin{array}{l}\text { ABBPPST (CERN-BEBC) } \\
\text { (deuterium) }\end{array}$ & $\begin{array}{l}r_{v}=0.06 \pm 0.06 \\
(0.06)\end{array}$ & $\begin{array}{l}r_{\bar{v}}=0.02 \pm 0.09 \\
(-0.01)\end{array}$ & 48 \\
\hline
\end{tabular}

scattering. Each group has extracted its most precise value of $\sin ^{2} \theta_{W}$ from the ratio $\sigma_{\bar{v}_{\mu} e} / \sigma_{v_{\mu} e}$, in which many of the systematic uncertainties cancel. We have used the individual cross sections (which are more useful for searching for deviations from the standard model) but have taken the correlations in the systematic uncertainties properly into account. Appropriate acceptance corrections are applied for both experiments. The updated Columbia-Brookhaven $v_{\mu} e$ cross section and five earlier $v_{\mu} e$ results ${ }^{71}$ are included as well.

We have computed new theoretical expressions for the reactor $\bar{v}_{e} e \rightarrow \bar{v}_{e} e$ experiment using a more accurate $\bar{v}_{e}$ spectrum $^{72,73}$ than the one used in earlier analyses, and have folded a $15 \%$ flux uncertainty (partially correlated between the two $E_{e}$ bins) into the expressions.
Experimental results on atomic parity violation ${ }^{74-81}$ are listed in Table $\mathrm{V}$, along with the extracted weak charge

$$
Q_{W}=-2\left[C_{1 u}(2 Z+N)+C_{1 d}(Z+2 N)\right] .
$$

The cesium results from Paris and Boulder are very precise and the theoretical uncertainties ${ }^{10,82}$ (from the atomic wave functions) are quite small. The theoretical uncertainties are $100 \%$ correlated between the Paris and Boulder cesium experiments and also between the Moscow and Oxford bismuth experiments.

Asymmetries in polarized $l^{ \pm} N$ scattering, ${ }^{83,84}$ where $l=\mu$ or $e$, are listed in Table VI. A $7 \%$ theoretical uncertainty has been folded into the theoretical expression for the SLAC $e \mathrm{D}$ asymmetry.

TABLE III. Results for ${ }^{(-)} p \rightarrow \stackrel{(-)}{v}^{v}$ elastic scattering and coherent pion production $v N \rightarrow v \pi^{0} N$. $\beta \equiv \epsilon_{L}(u)-\epsilon_{R}(u)-\epsilon_{L}(d)+\epsilon_{R}(d)$ is predicted to be 1.00 in the standard model.

\begin{tabular}{llc}
\hline \multicolumn{1}{c}{ Group } & \multicolumn{1}{c}{ Experimental value } & Reference \\
\hline E734 (BNL) & $\frac{d \sigma}{d Q^{2}}(v p \rightarrow v p)$ & 50 \\
$\left(8 Q^{2}\right.$ values $)$ & $\frac{d \sigma}{d Q^{2}}(\bar{v} p \rightarrow \bar{v} p)$ & \\
$\begin{array}{l}\text { SKAT (Serpukhov) } \\
\text { coherent } \pi^{0}\end{array}$ & $|\beta|=0.99 \pm 0.20$ & 51 \\
$\begin{array}{l}\text { CB (Fermilab) } \\
\text { coherent } \pi^{0}\end{array}$ & $|\beta|=0.98 \pm 0.24$ & 52 \\
$\begin{array}{l}\text { CHARM (CERN) } \\
\text { coherent } \pi^{0}\end{array}$ & $|\beta|=1.08 \pm 0.24$ & 53 \\
$\begin{array}{l}\text { AP (CERN) } \\
\text { coherent } \pi^{0}\end{array}$ & $|\beta|=0.93 \pm 0.16$ & 54 \\
\hline \hline
\end{tabular}


TABLE IV. Results for $\stackrel{(-)}{v} e \rightarrow \stackrel{(-)}{v} e$ elastic scattering. Except for the UCI reactor experiment all $\sigma / E$ are in units of $10^{-42}$ $\mathrm{cm}^{2} / \mathrm{GeV}$. The two uncertainties for the E734 and CHARM experiments are statistical and systematic, respectively, with the systematic uncertainties strongly correlated between $\sigma_{v_{\mu} e}$ and $\sigma_{\bar{v}_{\mu} e}$. The numbers in parentheses are the predictions of the standard model for $\sin ^{2} \theta_{W}=0.230$.

\begin{tabular}{|c|c|c|c|}
\hline Group & Experimental & lue & Reference \\
\hline E734 (BNL) & $\begin{array}{l}\sigma_{v_{\mu} e} / E_{v}=1.60 \pm 0.29 \pm 0.27 \\
(1.55)\end{array}$ & $\begin{array}{l}\sigma_{\bar{v}_{\mu} e^{e}} / E_{\bar{v}}=1.16 \pm 0.20 \pm 0.14 \\
(1.33)\end{array}$ & 62 \\
\hline CHARM (CERN) & $\begin{array}{l}\sigma_{v_{\mu} e} / E_{v}=1.9 \pm 0.4 \pm 0.4 \\
(1.6)\end{array}$ & $\begin{array}{l}\sigma_{\bar{v}_{\mu} e^{e}} / E_{\bar{v}}=1.5 \pm 0.3 \pm 0.4 \\
(1.3)\end{array}$ & 63 \\
\hline $\begin{array}{l}\text { CB }(\text { Fermilab } 15 \mathrm{ft}) \\
\text { (preliminary) }\end{array}$ & $\begin{array}{l}\sigma_{v_{\mu} e} / E_{v}=1.52 \pm 0.39 \\
(1.59)\end{array}$ & & 64 \\
\hline VMWOF (Fermilab) & $\begin{array}{l}\sigma_{v_{\mu}{ }^{e}} / E_{v}=1.40 \pm 0.50 \\
(1.59)\end{array}$ & & 65 \\
\hline GGM (CERN) & $\begin{array}{l}\sigma_{v_{\mu} e} / E_{v}=2.4_{-0.9}^{+1.2} \\
(1.6)\end{array}$ & & 66 \\
\hline AP (CERN) & $\begin{array}{l}\sigma_{v_{\mu} e} / E_{v}=1.1 \pm 0.6 \\
(1.6)\end{array}$ & $\begin{array}{l}\sigma_{\bar{v}_{\mu}{ }^{e}} / E_{\bar{v}}=2.2 \pm 1.0 \\
(1.3)\end{array}$ & 67 \\
\hline GGM (CERN) & & $\begin{array}{l}\sigma_{\bar{v}_{\mu} e^{e}} / E_{\bar{v}}=1.0_{-0.6}^{+1.3} \\
(1.3)\end{array}$ & 68 \\
\hline $\begin{array}{l}\text { UCI (Savannah River Reactor) } \\
1.5<E_{e}<3.0 \mathrm{MeV}\end{array}$ & $\begin{array}{l}\sigma_{\bar{v}_{e} e}=(4.6 \pm 1.3) \times 10^{-45} \mathrm{~cm}^{2} / \text { fission } \\
(3.33)\end{array}$ & & 69 \\
\hline $3.0<E_{e}<4.5 \mathrm{MeV}$ & $\begin{array}{l}\sigma_{\bar{v}_{e} e^{e}}=(1.12 \pm 0.29) \times 10^{-45} \mathrm{~cm}^{2} / \text { fission } \\
(0.55)\end{array}$ & & \\
\hline ILM (LANL) & $\begin{array}{l}\sigma_{v_{e} e} / E_{v}=8.9 \pm 3.5 \\
(9.4)\end{array}$ & & 70 \\
\hline
\end{tabular}

The $W$ and $Z$ mass results ${ }^{85,86}$ from UA1 and UA2 are listed in Table VII.

Forward-backward asymmetries $\left(A_{l l}\right)$ and total cross sections $R_{l l}$ (relative to pure QED) for $e^{+} e^{-} \rightarrow l^{+} l^{-}$are listed $^{87-103}$ in Tables VIII-XI. The numbers have been corrected for pure QED effects by the experimental groups. Asymmetries in $e^{+} e^{-} \rightarrow e^{+} e^{-}, c \bar{c}$, and $b \bar{b}$ and constraints from the $e^{+} e^{-} \rightarrow$ hadrons total cross section are consistent with the standard model and will be discussed in our longer paper. ${ }^{17}$

\section{RESULTS}

\section{A. $\sin ^{2} \theta_{W}$}

The values of $\sin ^{2} \theta_{W}$ determined from various reactions and from a global fit to all data are listed in Table XII. For many of the individual reactions the experimental and theoretical uncertainties ${ }^{104}$ are displayed separately. The central values for all fits assume $m_{t}=45$ $\mathrm{GeV}$ and $M_{H}=100 \mathrm{GeV}$, while the theoretical uncertainties assume $m_{t} \leq 100 \mathrm{GeV}, M_{H} \leq 1 \mathrm{TeV}$, three fermion families, and reasonable ranges for all other parameters. The theoretical uncertainties for deep-inelastic scattering are broken down in Table XIII.

The $\sin ^{2} \theta_{W}$ values for the various reactions are in remarkable agreement, indicating the success of the standard model. This is shown in Fig. 1(a), in which the various $\sin ^{2} \theta_{W}$ values are displayed as a function of the

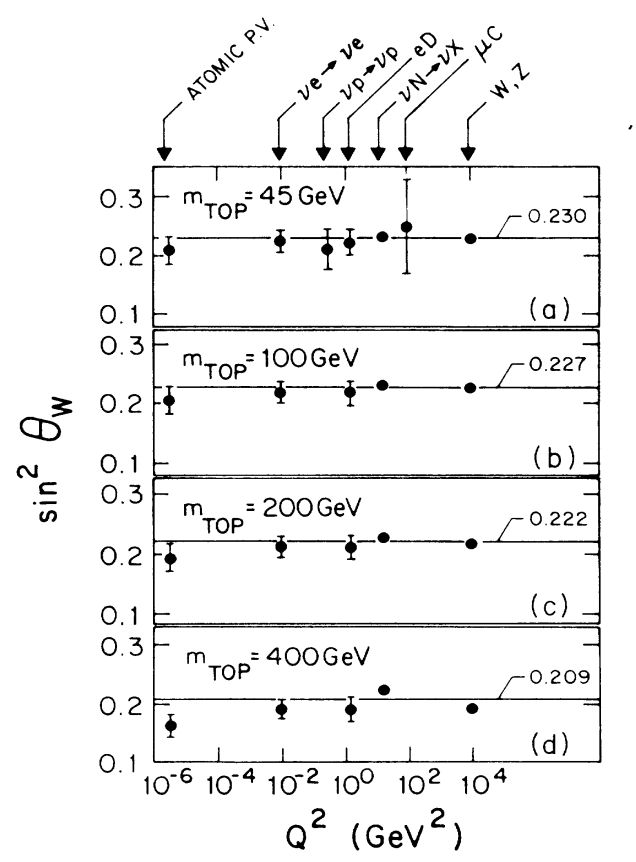

FIG. 1. (a) $\sin ^{2} \theta_{W}$ for various reactions as a function of the typical $Q^{2}$, determined for $m_{t}=45 \mathrm{GeV}$. The best-fit line $\sin ^{2} \theta_{W}=0.230$ is also shown. (b) $-(\mathrm{d}) \sin ^{2} \theta_{W}$ values determined for $m_{t}=100,200$, and $400 \mathrm{GeV}$. 
TABLE V. Experimental results on atomic parity violation and the extracted weak charge $Q_{W}$. The two errors in $Q_{W}$ are experimental and theoretical (Refs. 10 and 82), respectively. The numbers in parentheses are the expectations of the standard model for $\sin ^{2} \theta_{W}=0.230$.

\begin{tabular}{|c|c|c|c|}
\hline Group & Experimental value & $-Q_{W}(N, Z)$ & Reference \\
\hline \multicolumn{4}{|l|}{ Cesium } \\
\hline Paris (1986) & $\operatorname{Im} E_{1}^{P V} / \beta=-1.52 \pm 0.18 \mathrm{mV} / \mathrm{cm}$ & $\begin{array}{l}68 \pm 9 \pm 3 \\
(71.8)\end{array}$ & 74 \\
\hline Boulder (1985) & $-1.65 \pm 0.13 \mathrm{mV} / \mathrm{cm}$ & $\begin{array}{l}74 \pm 6 \pm 3 \\
(71.8)\end{array}$ & 75 \\
\hline \multicolumn{4}{|l|}{ Thallium } \\
\hline Berkeley (1984) & $\operatorname{Im} E_{1}^{P V} / \beta=-1.73 \pm 0.33 \mathrm{mV} / \mathrm{cm}$ & $\begin{array}{l}164 \pm 31 \pm 50 \\
(113.7)\end{array}$ & 76 \\
\hline \multicolumn{4}{|l|}{ Lead } \\
\hline Seattle (1984) & $10^{8} \operatorname{Im} E_{1}^{P V} / M_{1}=-9.9 \pm 2.5$ & $\begin{array}{l}84 \pm 21 \pm 13 \\
(115.6)\end{array}$ & 77 \\
\hline \multicolumn{4}{|l|}{ Bismuth $(876 \mathrm{~nm})$} \\
\hline Seattle (1984) & $10^{8} \operatorname{Im} E_{1}^{P V} / M_{1}=-10.4 \pm 1.7$ & $\begin{array}{l}116 \pm 19 \pm 29 \\
(116.5)\end{array}$ & 78 \\
\hline \multicolumn{4}{|l|}{ Bismuth $(648 \mathrm{~nm})$} \\
\hline Moscow (1984) & $10^{8} \operatorname{Im} E_{1}^{P V} / M_{1}=-7.8 \pm 1.8$ & $\begin{array}{l}70 \pm 16 \pm 17 \\
(116.5)\end{array}$ & 79 \\
\hline $\begin{array}{l}\text { Oxford (1984) } \\
\text { (preliminary) }\end{array}$ & $-9.3 \pm 1.5$ & $\begin{array}{l}83 \pm 13 \pm 20 \\
(116.5)\end{array}$ & 80 \\
\hline
\end{tabular}

typical $Q^{2}$ values of the reactions.

The best fit to all data yields $\sin ^{2} \theta_{W}=0.230 \pm 0.0048$. As can be seen from Tables I-XI, all data are in excellent agreement with the standard model for this value of $\sin ^{2} \theta_{W}$. The $\chi^{2}$ per degree of freedom (145/184) is slightly low (possibly due to conservative overestimates of systematic errors or to correlations between the various experiments that have not been taken into account), but nevertheless reasonable. The last entry (All data) in Table XII includes certain reactions (e.g., deep-inelastic scattering from nonisoscalar targets and $\left.v_{e} e \rightarrow v_{e} e\right)$ that are less precise than those listed explicitly in Table XII. Our rationale for including these results is that they will be useful in studies of limits on deviations from the standard model, and it is desirable to use the same data sets for all global fits. It is reassuring, however, that reasonable variations on the data set yield consistent results. For example, the first six reactions in Table XII plus $e^{+} e^{-}$data yield $\sin ^{2} \theta_{W}=0.228 \pm 0.0048$ while a fit to only the most precise experiments of each type yields $0.230 \pm 0.0051$. Similarly, the four new deep-inelastic experiments give $\sin ^{2} \theta_{W}=0.234 \pm 0.007$, compared to the value $0.233 \pm 0.006$ obtained when the older experiments are included. In contrast, the CHARM and BNL E734 $\stackrel{(-)}{v}_{\mu} e$ experiments yield the average $0.213 \pm 0.022$. This is pulled up by $\simeq \frac{1}{2} \sigma$ (to $0.223 \pm 0.019)$ by the other experiments listed in Table IV.

We do not quote a $\sin ^{2} \theta_{W}$ for $e^{+} e^{-}$reactions. This is because at PEP and PETRA energies the asymmetries $A_{l l}$ are nearly an absolute prediction of the standard model; i.e., they are practically independent of the values of the standard-model parameters. The cross-section ratios $R_{l l}$ are predicted to be close to unity and change by only a few percent for a wide range of $\sin ^{2} \theta_{W}$. Therefore, all values of $\sin ^{2} \theta_{W}$ from 0.1 to 0.4 give a good description of the data, and to first approximation the $\chi^{2}$ distribution is nearly flat for $\sin ^{2} \theta_{W}$ in that range. A

TABLE VI. Polarized $l^{ \pm} N$ scattering. $b$ is a measure of the $\sigma_{\mu^{+} C}\left(\lambda=-\frac{1}{2}\right)-\sigma_{\mu^{-} C^{-}}\left(\lambda=+\frac{1}{2}\right)$ asymmetry. The numbers in parentheses are the predictions of the standard model for $\sin ^{2} \theta_{W}=0.230$.

\begin{tabular}{clc}
\hline \multicolumn{1}{c}{ Group } & \multicolumn{1}{c}{ Experimental value } & Reference \\
\hline$e \mathrm{D}$ (SLAC) & $\begin{array}{l}\text { Polarization asymmetries } \\
\left(11 Q^{2} \text { values }\right)\end{array}$ & 83 \\
BCDMS $\left(\mu^{ \pm} \mathrm{C}\right)(\mathrm{CERN})$ & $\begin{array}{l}(-0.147 \pm 0.044) \times 10^{-3} \mathrm{GeV}^{-2} \\
\left(-0.144 \times 10^{-3}\right)\end{array}$ & 84 \\
$E_{\mu}=200 \mathrm{GeV}$ & $b=(-0.174 \pm 0.080) \times 10^{-3} \mathrm{GeV}^{-2}$ & \\
$E_{\mu}=120 \mathrm{GeV}$ & $\left(-0.145 \times 10^{-3}\right)$ & \\
\hline \hline
\end{tabular}


TABLE VII. The $W$ and $Z$ masses (in GeV). The first uncertainties are mainly statistical and the second are energy calibration uncertainties that are $100 \%$ correlated between $M_{W}$ and $M_{Z}$ for each group. The numbers in parentheses are the standard-model predictions for the global best-fit value $\sin ^{2} \theta_{W}=0.230$. The last two rows are predictions of the standard model, using $\sin ^{2} \theta_{W}$ determined from deep-inelastic scattering, with and without radiative corrections, respectively.

\begin{tabular}{|c|c|c|c|}
\hline Group & $M_{W}$ & $M_{Z}$ & Reference \\
\hline UA2 (CERN) & $\begin{array}{l}80.2 \pm 0.8 \pm 1.3 \\
(80.7)\end{array}$ & $\begin{array}{l}91.5 \pm 1.2 \pm 1.7 \\
(91.9)\end{array}$ & 85 \\
\hline UA1 (CERN) & $\begin{array}{l}83.5_{-1.0}^{+1.1} \pm 2.7 \\
(80.7)\end{array}$ & $\begin{array}{l}93.0 \pm 1.4 \pm 3.0 \\
(91.9)\end{array}$ & 86 \\
\hline UA $1+$ UA2 combined & $80.9 \pm 1.4$ & $91.9 \pm 1.8$ & \\
\hline $\begin{array}{l}\text { Prediction from deep inelastic } \\
\quad \text { (with radiative corrections; } \\
\sin ^{2} \theta_{W}=0.233 \pm 0.006 \text { ) }\end{array}$ & $80.2 \pm 1.1$ & $91.6 \pm 0.9$ & \\
\hline $\begin{array}{l}\text { Prediction from deep inelastic } \\
\text { (without radiative corrections; } \\
\sin ^{2} \theta^{0}=0.242 \pm 0.006, \Delta r=\Delta s^{2}=0 \text { ) }\end{array}$ & $75.9 \pm 1.0$ & $87.1 \pm 0.7$ & \\
\hline
\end{tabular}

closer examination of the $\chi^{2}$ distribution reveals two shallow minima at $\sin ^{2} \theta_{W}=0.16_{-0.03}^{+0.04}$ and $\sin ^{2} \theta_{W}$ $=0.36 \pm 0.02$, with $\sin ^{2} \theta_{W}=0.23$ located on the edge of the lower minimum. This structure is due to a slight tendency for the $R_{l l}$ values in Tables IX and XI to decrease with energy. Such a decrease, if significant, can only be achieved if $\sin ^{2} \theta_{W}$ deviates significantly from $\frac{1}{4}$. However, we do not think that these values of $\sin ^{2} \theta_{W}$ constitute compelling evidence for a deviation from the standard model, because of the following reasons. (a) The error bars on these minima (defined by an increase $\Delta \chi^{2}=1$ from the value at the local minimum) cannot be

TABLE VIII. Forward-backward asymmetries $A_{\mu \mu}$ for $e^{+} e^{-} \rightarrow \mu^{+} \mu^{-}$. The theoretical prediction is nominally for $M_{Z}=91.9 \mathrm{GeV}$ and $\sin ^{2} \theta_{W}=0.230$, but in fact is almost independent of $M_{Z}$ and $\sin ^{2} \theta_{W}$ at PEP and PETRA energies. The PLUTO group has applied full electroweak radiative corrections to their $\mu$ asymmetry. We have renormalized their value of $A_{\mu \mu}=-14 \%$ to $-13 \%$, which corresponds to our theoretical expressions.

\begin{tabular}{|c|c|c|c|c|}
\hline Group & $\sqrt{s}(\mathrm{GeV})$ & $A_{\mu \mu}(\%)$ & $A_{\mu \mu}$ (theory) & Reference \\
\hline \multirow[t]{3}{*}{ CELLO } & 34.2 & $-6.4 \pm 6.4$ & -8.5 & 87 \\
\hline & 39.0 & $-4.8 \pm 6.5 \pm 1.0$ & -11.5 & \\
\hline & 44.0 & $-18.8 \pm 4.5 \pm 1.0$ & -15.5 & \\
\hline \multirow[t]{5}{*}{ JADE } & 13.9 & $2.7 \pm 4.9 \pm 1.0$ & -1.2 & 88 \\
\hline & 22.0 & $-10.6 \pm 6.4 \pm 1.0$ & -3.2 & \\
\hline & 34.4 & $-11.1 \pm 1.8 \pm 1.0$ & -8.6 & \\
\hline & 38.0 & $-9.7 \pm 5.0 \pm 1.0$ & -10.8 & \\
\hline & 43.7 & $-19.1 \pm 2.8 \pm 1.0$ & -15.3 & \\
\hline \multirow[t]{9}{*}{ Mark J } & 14.0 & $+5.3 \pm 5.0 \pm 0.5$ & -1.3 & 89 \\
\hline & 22.5 & $-4.3 \pm 6.1 \pm 0.5$ & -3.4 & \\
\hline & 34.8 & $-10.4 \pm 1.3 \pm 0.5$ & -8.8 & \\
\hline & 36.4 & $-13.6 \pm 13.5 \pm 0.5$ & -9.8 & \\
\hline & 38.3 & $-12.3 \pm 5.3 \pm 0.5$ & -11.0 & \\
\hline & 40.4 & $+5.0 \pm 10.5 \pm 0.5$ & -12.6 & \\
\hline & 42.0 & $-15.9 \pm 9.3 \pm 0.5$ & -13.8 & \\
\hline & 43.8 & $-15.6 \pm 3.0 \pm 0.5$ & -15.4 & \\
\hline & 46.1 & $-17.6 \pm 8.3 \pm 0.5$ & -17.5 & \\
\hline PLUTO & 34.7 & $-13.0 \pm 3.2 \pm 1.0$ & -8.8 & 90 \\
\hline \multirow[t]{5}{*}{ TASSO } & 13.9 & $-1.0 \pm 6.0$ & -1.2 & 91 \\
\hline & 22.3 & $-13.0 \pm 7.0$ & -3.3 & \\
\hline & 34.8 & $-9.6 \pm 1.5 \pm 0.5$ & -8.8 & \\
\hline & 38.3 & $+2.4 \pm 8.6 \pm 0.5$ & -11.0 & \\
\hline & 43.6 & $-17.3 \pm 4.3 \pm 0.5$ & -15.2 & \\
\hline HRS & 29.0 & $-4.9 \pm 1.5 \pm 0.5$ & -5.8 & 92 \\
\hline Mark II & 29.0 & $-7.1 \pm 1.7$ & -5.8 & 93 \\
\hline MAC & 29.0 & $-5.9 \pm 0.8 \pm 0.2$ & -5.8 & 94 \\
\hline
\end{tabular}


TABLE IX. Cross sections $R_{\mu \mu}$ (relative to QED) for $e^{+} e^{-} \rightarrow \mu^{+} \mu^{-}$. We assume $100 \%$ correlation of the systematic errors within each experiment. In several cases we have combined the data in nearby energy bins.

\begin{tabular}{|c|c|c|c|c|}
\hline Group & $\sqrt{s}(\mathrm{GeV})$ & $R_{\mu \mu}(\%)$ & $R_{\mu \mu}$ (theory) & Reference \\
\hline \multirow[t]{7}{*}{ CELLO } & 14.0 & $1.16 \pm 0.08 \pm 0.05$ & 1.000 & 87 \\
\hline & 22.2 & $1.00 \pm 0.08 \pm 0.05$ & 1.000 & \\
\hline & 34.0 & $0.89 \pm 0.06 \pm 0.05$ & 1.002 & \\
\hline & 38.2 & $0.96 \pm 0.07 \pm 0.04$ & 1.005 & \\
\hline & 41.3 & $1.06 \pm 0.17 \pm 0.04$ & 1.007 & \\
\hline & 43.8 & $0.985 \pm 0.055 \pm 0.04$ & 1.010 & \\
\hline & 46.2 & $0.95 \pm 0.22 \pm 0.04$ & 1.013 & \\
\hline \multirow[t]{8}{*}{ JADE } & 13.8 & $1.01 \pm 0.05 \pm 0.05$ & 1.000 & 88 \\
\hline & 22.3 & $1.03 \pm 0.06 \pm 0.05$ & 1.000 & \\
\hline & 31.6 & $1.10 \pm 0.08 \pm 0.05$ & 1.002 & \\
\hline & 34.6 & $0.991 \pm 0.018 \pm 0.05$ & 1.003 & \\
\hline & 37.6 & $1.12 \pm 0.12 \pm 0.05$ & 1.004 & \\
\hline & 41.3 & $0.97 \pm 0.06 \pm 0.05$ & 1.007 & \\
\hline & 44.2 & $0.98 \pm 0.05 \pm 0.05$ & 1.010 & \\
\hline & 46.0 & $1.03 \pm 0.09 \pm 0.05$ & 1.013 & \\
\hline \multirow[t]{9}{*}{ Mark J } & 14.0 & $1.04 \pm 0.05 \pm 0.04$ & 1.000 & 89 \\
\hline & 22.5 & $1.02 \pm 0.05 \pm 0.04$ & 1.000 & \\
\hline & 34.8 & $0.99 \pm 0.012 \pm 0.03$ & 1.003 & \\
\hline & 36.4 & $1.08 \pm 0.13 \pm 0.03$ & 1.004 & \\
\hline & 38.3 & $1.07 \pm 0.05 \pm 0.03$ & 1.005 & \\
\hline & 40.4 & $0.93 \pm 0.10 \pm 0.03$ & 1.006 & \\
\hline & 42.0 & $1.04 \pm 0.09 \pm 0.03$ & 1.008 & \\
\hline & 43.8 & $0.99 \pm 0.03 \pm 0.03$ & 1.010 & \\
\hline & 46.1 & $0.96 \pm 0.08 \pm 0.03$ & 1.013 & \\
\hline PLUTO & 34.7 & $0.94 \pm 0.03 \pm 0.04$ & 1.003 & 95 \\
\hline \multirow[t]{3}{*}{ TASSO } & 34.5 & $1.002 \pm 0.020 \pm 0.035$ & 1.003 & 96 \\
\hline & 38.3 & $0.889 \pm 0.068 \pm 0.052$ & 1.005 & \\
\hline & 43.6 & $0.894 \pm 0.037 \pm 0.048$ & 1.009 & \\
\hline HRS & 29.0 & $0.990 \pm 0.017 \pm 0.030$ & 1.001 & 92 \\
\hline Mark II & 29.0 & $1.002 \pm 0.013 \pm 0.016$ & 1.001 & 93 \\
\hline MAC & 29.0 & $1.01 \pm 0.01 \pm 0.03$ & 1.001 & 94 \\
\hline
\end{tabular}

interpreted as ordinary $1 \sigma$ uncertainties. This is because the $\chi^{2}$ distribution is highly non-Gaussian, and $\sin ^{2} \theta_{W}=0.23$ is only $\Delta \chi^{2}=1.7$ above the minimum value, i.e., within the $80 \%$-confidence-level interval. (b) The decrease in $R_{l l}$ is of the same order of magnitude as the systematic errors in the individual experiments. We have assumed that the systematic errors for each experiment are normalization errors independent of energy, but we do not have sufficient knowledge of (or limits on) possible energy-dependent systematic effects to exclude them as the origin of the decrease of the $R_{l l}$.

\section{B. $\rho$ and $\sin ^{2} \theta_{W}$}

The quantity $\rho \equiv M_{W}{ }^{2} /\left(M_{Z}{ }^{2} \cos ^{2} \theta_{W}\right)$ is exactly one (in our renormalization scheme) in the standard model or any $\mathbf{S U}(2) \times \mathbf{U}(1)$ model in which all symmetry breaking is due to Higgs doublets. ${ }^{105}$ However, $\rho \neq 1$ can occur if there are Higgs multiplets with $I>\frac{1}{2}$ with significant vacuum expectation values (VEV's). One has

$$
\rho=\frac{\sum_{i}\left(I_{i}{ }^{2}-I_{3 i}{ }^{2}+I_{i}\right)\left\langle\phi_{i}\right\rangle^{2}}{\sum_{i} 2 I_{3 i}{ }^{2}\left\langle\phi_{i}\right\rangle^{2}},
$$

where $\left\langle\phi_{i}\right\rangle$ is the VEV of a Higgs field $\phi_{i}$ with weak isospin and $z$ component $I_{i}$ and $I_{3 i}$, respectively. $\rho \neq 1$ can also be generated by mixing with additional $Z$ bosons, but that implies other effects as well and will be treated separately. For $\rho \neq 1$, the weak amplitudes in Sec. II are multiplied by $\rho$, the formula for $\boldsymbol{M}_{Z}$ is divided by $\sqrt{\rho}$, and the $\boldsymbol{M}_{\boldsymbol{W}}$ formula is unchanged.

Fits to $\rho$ and $\sin ^{2} \theta_{W}$ are presented in Table XIV, and the various theoretical uncertainties for deep-inelastic scattering are detailed in Table XIII. One finds ${ }^{106}$ $\rho=0.998 \pm 0.0086$ and $\sin ^{2} \theta_{W}=0.229 \pm 0.0064$, remarkably consistent with $\rho=1$. From (15) this implies $90 \%$ confidence-level (C.L.) limits

$$
\frac{\left|\left\langle\phi_{10}\right\rangle\right|}{\left|\left\langle\phi_{1 / 21 / 2}\right\rangle\right|}<0.047
$$

and

$$
\frac{\left|\left\langle\phi_{1 \pm 1}\right\rangle\right|}{\left|\left\langle\phi_{1 / 21 / 2}\right\rangle\right|}<0.081
$$

on the VEV's of Higgs triplets with $I_{3}=0$ or \pm 1 , respectively, relative to that of the ordinary Higgs doublet (these limits assume that only one new VEV is present so that there are no cancellations). The allowed region ${ }^{107}$ 
TABLE X. Forward-backward asymmetries $A_{\tau \tau}$ for $e^{+} e^{-} \rightarrow \tau^{+} \tau^{-}$. We have renormalized the PLUTO asymmetry from $-5.9 \%$ to account for the different radiative corrections that have been applied.

\begin{tabular}{lcrcc}
\hline \hline Group & $\sqrt{s}(\mathrm{GeV})$ & \multicolumn{1}{c}{$A_{\tau \tau}(\%)$} & $A_{\tau \tau}$ (theory) & Reference \\
\hline CELLO & 34.2 & $-10.3 \pm 5.2$ & -8.5 & 97 \\
& 39.0 & $-13.5 \pm 6.9 \pm 2.0$ & -11.5 & \\
JADE & 44.0 & $-22.3 \pm 4.1 \pm 2.0$ & -15.5 & 98 \\
& 34.6 & $-6.0 \pm 2.5 \pm 1.0$ & -8.7 & \\
Mark J & 38.0 & $+7.5 \pm 6.3 \pm 1.0$ & -10.8 & 99 \\
& 43.7 & $-17.0 \pm 3.6 \pm 1.0$ & -15.3 & 100 \\
PLUTO & 35.0 & $-8.6 \pm 3.7 \pm 1.5$ & -8.9 & 91 \\
TASSO & 43.9 & $-12.8 \pm 7.0 \pm 1.5$ & -15.4 & \\
& 34.6 & $-5.2 \pm 6.8+0.5$ & -8.7 & 101 \\
HRS & 13.9 & $+15 \pm 12$ & -1.2 & 93 \\
Mark II & 22.3 & $0 \pm 10$ & -3.3 & 9.6 \\
MAC & 34.5 & $-4.9 \pm 5.3 \pm 1.2$ & -5.8 & -5.8 \\
\hline \hline
\end{tabular}

in the $\rho-\sin ^{2} \theta_{W}$ plane is shown in Fig. 2, and the regions allowed by various individual reactions are shown in Fig. $3(\mathrm{a})$.

The allowed region for $e^{+} e^{-}$data are shown for various confidence levels in Fig. 3(b). Just as in the $\sin ^{2} \theta_{W}$ fit, the $R_{l l}$ values lead to two minima. The standardmodel point $\rho=1, \sin ^{2} \theta_{W}=0.23$ is on the edge of the low $\sin ^{2} \theta_{W}$ minimum, with $\Delta \chi^{2}=5.6$ above the minimum value. It is therefore within the $95 \%$-C.L. contour for the $e^{+} e^{-}$data. Again the small discrepancy is consistent with a fluctuation or with possible energy- dependent systematic effects, and the evidence is not strong enough to claim that there is new physics beyond the standard model. However, a possible explanation involving new physics is discussed below in Sec. IV F.

The $e, \mu$, and $\tau$ vector couplings are close to zero; hence, they and $\sin ^{2} \theta_{W}$ are not well determined by the $e^{+} e^{-}$data. However, if $\sin ^{2} \theta_{W}$ is fixed at the canonical value 0.23 found from other reactions, then $\rho$ is determined rather well, mainly by the forward-backward asymmetries $A_{l l}$. One obtains $\rho=1.039 \pm 0.043$, essentially independent of $\sin ^{2} \theta_{W}$ for reasonable values.

TABLE XI. Total cross section $R_{\tau \tau}$ (relative to QED) for $e^{+} e^{-} \rightarrow \tau^{+} \tau^{-}$. We assume $100 \%$ correlation of systematic errors within each experiment.

\begin{tabular}{|c|c|c|c|c|}
\hline Group & $\sqrt{s}(\mathrm{GeV})$ & $R_{\tau \tau}(\%)$ & $R_{\tau \tau}$ (theory) & Reference \\
\hline \multirow[t]{3}{*}{ CELLO } & 34.2 & $1.03 \pm 0.05 \pm 0.07$ & 1.003 & 102 \\
\hline & 39.0 & $1.114 \pm 0.076 \pm 0.060$ & 1.005 & \\
\hline & 44.0 & $1.004 \pm 0.041 \pm 0.052$ & 1.010 & \\
\hline \multirow[t]{5}{*}{ JADE } & 12.0 & $1.29 \pm 0.24 \pm 0.20$ & 1.000 & 98 \\
\hline & 25.6 & $1.16 \pm 0.16 \pm 0.11$ & 1.001 & \\
\hline & 30.6 & $1.06 \pm 0.10 \pm 0.08$ & 1.001 & \\
\hline & 34.6 & $0.959 \pm 0.019 \pm 0.033$ & 1.003 & \\
\hline & 43.1 & $0.980 \pm 0.037 \pm 0.041$ & 1.009 & \\
\hline \multirow[t]{6}{*}{ Mark J } & 14.1 & $1.04 \pm 0.14 \pm 0.04$ & 1.000 & 99 \\
\hline & 22.4 & $1.08 \pm 0.11 \pm 0.03$ & 1.000 & \\
\hline & 34.6 & $0.965 \pm 0.033 \pm 0.03$ & 1.003 & \\
\hline & 40.8 & $0.93 \pm 0.09 \pm 0.03$ & 1.006 & \\
\hline & 44.2 & $1.16 \pm 0.10 \pm 0.03$ & 1.010 & \\
\hline & 46.1 & $1.05 \pm 0.16 \pm 0.03$ & 1.013 & \\
\hline PLUTO & 34.7 & $0.89 \pm 0.05 \pm 0.08$ & 1.003 & 100 \\
\hline \multirow[t]{4}{*}{ TASSO } & 13.9 & $1.04 \pm 0.13$ & 1.000 & 96 \\
\hline & 22.3 & $1.03 \pm 0.17$ & 1.000 & \\
\hline & 34.5 & $1.03 \pm 0.05_{-0.11}^{+0.06}$ & 1.003 & \\
\hline & 43.1 & $1.05 \pm 0.17$ & 1.009 & \\
\hline HRS & 29.0 & $1.10 \pm 0.03 \pm 0.04$ & 1.001 & 101 \\
\hline Mark II & 29.0 & $0.996 \pm 0.016 \pm 0.028$ & 1.001 & 93 \\
\hline MAC & 29.0 & $0.98 \pm 0.01 \pm 0.034$ & 1.001 & 103 \\
\hline
\end{tabular}


TABLE XII. Determination of $\sin ^{2} \theta_{W}$ from various reactions. Where two errors are shown the first is experimental and the second (in square brackets) is theoretical. In the other cases the theoretical and experimental uncertainties are combined.

\begin{tabular}{crl}
\hline \hline Reaction & \multicolumn{1}{c}{$\sin ^{2} \theta_{W}$} & \multicolumn{1}{c}{$\chi^{2} / \mathrm{DF}$} \\
\hline Deep inelastic (isoscalar) & $0.233 \pm 0.003 \pm[0.005]$ & $15.1 / 20$ \\
$(-),(-))$ & $0.210 \pm 0.033$ & $13.1 / 14$ \\
$v{ }_{\mu} p \rightarrow v{ }_{\mu} p$ & $0.223 \pm 0.018 \pm[0.002]$ & $4.1 / 11$ \\
$(-)_{\mu} e \rightarrow v{ }_{\mu} e$ & $0.228 \pm 0.007 \pm[0.002]$ & $1.9 / 3$ \\
$W, Z$ & $0.209 \pm 0.018 \pm[0.014]$ & $5.9 / 6$ \\
Atomic parity violation & $0.221 \pm 0.015 \pm[0.013]$ & $10.4 / 10$ \\
SLAC $e \mathrm{D}$ & $0.25 \pm 0.08$ & $0.1 / 1$ \\
$\mu \mathrm{C}$ & $0.230 \pm 0.0048$ & $145 / 184$ \\
All data & & \\
\hline \hline
\end{tabular}

\section{Radiative corrections}

The data are now sufficiently precise to begin testing the standard model at the radiative correction level. A conventional measure of radiative corrections is the parameter $\Delta r$ defined in (13), which represents the radiative corrections relating $G_{F}$ (measured in muon decay) to the $W$ and $Z$ masses. A simultaneous fit of deep-inelastic (isoscalar-target) data and the $W$ and $Z$ masses to $\Delta r$ and $\sin ^{2} \theta_{W}$ yields the allowed region shown in Fig. 4. The corresponding value $\Delta r=0.077 \pm 0.037$ is in excellent

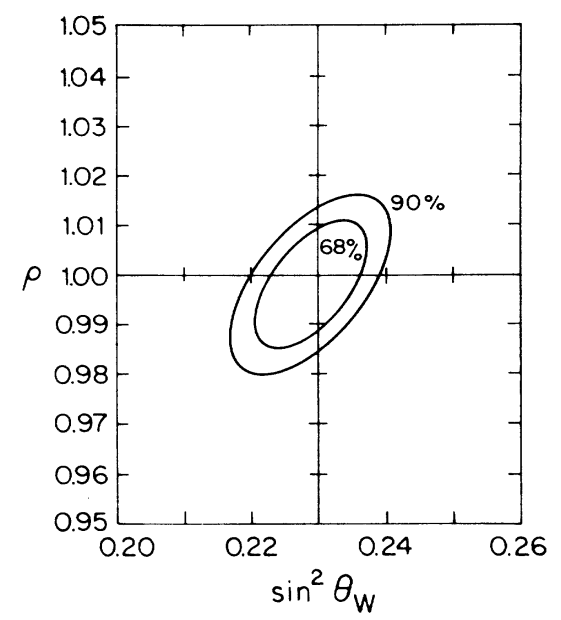

FIG. 2. The allowed regions in the $\sin ^{2} \theta_{W-\rho}$ plane at $90 \%$ C.L. and $68 \%$ C.L.

agreement with the value $0.0713 \pm 0.0013$ predicted for $m_{t}=45 \mathrm{GeV}$ and $M_{H}=100 \mathrm{GeV}$.

However, the $\Delta r-\sin ^{2} \theta_{W}$ fit is not the best test of radiative corrections, because the value of $\sin ^{2} \theta_{W}$ extracted from the data itself depends on the radiative corrections assumed. In particular, any noncanonical contribution to $\Delta r$ (such as a very large $m_{t}$ ) could also affect $\sin ^{2} \theta_{W}$. A better measure is obtained if the $M_{W}$ and $M_{Z}$ predictions are written in terms of

TABLE XIII. Theoretical uncertainties for deep-inelastic scattering. The third column refers to the $\sin ^{2} \theta_{W}$ fit with $\rho=1$, while the last two columns refer to the $\left(\rho, \sin ^{2} \theta_{W}\right)$ fit. $U \equiv \int_{0}^{1} x u\left(x, Q_{0}{ }^{2}\right) d x$ is the first moment of $u\left(x, Q^{2}\right)$ evaluated at $Q_{0}{ }^{2}=5 \mathrm{GeV}^{2} . U_{V}$ and $D_{V}$ refer to the valence-quark distributions, $\Lambda_{\mathrm{QCD}}$ is an effective leading-order QCD $\Lambda$ parameter, and $R_{L}=\left(F_{2}-2 x F_{1}\right) / 2 x F_{1}$ is the longitudinal structure function [replacing $R_{L}=0.1$ by a QCD-inspired $R_{L}\left(x, Q^{2}\right)$ changes $\sin ^{2} \theta_{W}$ by only + 0.0004]. Assuming three fermion families it is sufficient to parametrize the transitions between $u, d, c$, and $s$ by an effective Calibbo angle. For more than three families $\Delta \sin ^{2} \theta_{W} \mid$ кмс increases to $\left({ }_{-0.001}^{+0.008}\right)$, leading to a total theory error $\Delta \sin ^{2} \theta_{W}={ }_{-0.0055}^{+0.0096}$. All theory uncertainties except $(\bar{U}+\bar{D}) /(U+D)$ are computed with $r=\sigma_{\bar{v} N}^{\mathrm{CC}} / \sigma_{v N}^{\mathrm{CC}}$ fixed (to avoid double counting). Several additional small $(\leq 0.001)$ uncertainties (e.g., from the shapes of the quark distributions at $\left.Q_{0}{ }^{2}\right)$ are due almost entirely to the charm threshold and are not independent of $m_{c}$.

\begin{tabular}{ccccc}
\hline \hline Quantity & Value & $\Delta \sin ^{2} \theta_{W}(\rho=1)$ & $\Delta \sin ^{2} \theta_{W}$ & $\Delta \rho$ \\
\hline$(\bar{U}+\bar{D}) /(U+D)$ & $0.125 \pm 0.020$ & \pm 0.002 & \pm 0.008 & \pm 0.006 \\
$\bar{S} / \bar{U}$ & $0.46 \pm 0.10$ & \pm 0.0007 & \pm 0.0003 & $\mp 0.0004$ \\
$C / S$ & $0.15 \pm 0.15$ & \pm 0.0002 & & \\
$(\bar{S}-S) / 2 S$ & $0 \pm 0.1$ & \pm 0.0003 & & \\
$D_{V} / U_{V}$ & $0.39 \pm 0.06$ & \pm 0.0006 & $\mp 0.0001$ & $\mp 0.0007$ \\
angles & & & & \\
$(3$ families) & $U_{u d}=0.973 \pm 0.002$ & $\mp 0.0003$ & & \\
$m_{c}(\mathrm{GeV})$ & & & & \\
$\Lambda_{\mathrm{QCD}}(\mathrm{MeV})$ & $1.5 \pm 0.3$ & \pm 0.0041 & \pm 0.0005 & $\mp 0.004$ \\
$R_{L}$ & $250 \pm 100$ & $\mp 0.0001$ & & \\
$m_{t}(\mathrm{GeV})$ & $0.1 \pm 0.1$ & $\mp 0.001$ & $\mp 0.001$ & \pm 0.001 \\
$M_{H}(\mathrm{GeV})$ & $25-100$ & \pm 0.0012 & \pm 0.002 & \pm 0.0015 \\
Highayashi-Maskawa-Cabibbo (KMC) & $10-1000$ & \pm 0.0002 & \pm 0.001 & \pm 0.001 \\
Totwist & & \pm 0.001 & & \\
Ther & & \pm 0.002 & & \\
\hline \hline
\end{tabular}


TABLE XIV. Determination of $\rho$ and $\sin ^{2} \theta_{W}$ from various reactions. Where two errors are shown the first is experimental and the second (in square brackets) is theoretical.

\begin{tabular}{ccccc}
\hline \hline Reaction & $\sin ^{2} \theta_{W}$ & $\rho$ & Correlation & $\chi^{2} / \mathrm{DF}$ \\
\hline Deep inelastic (isoscalar) & $0.232 \pm 0.014 \pm[0.008]$ & $0.999 \pm 0.013 \pm[0.008]$ & 0.90 & $15.1 / 19$ \\
$(-))(-))$ & $0.205 \pm 0.041$ & $0.98 \pm 0.06 \pm[0.05]$ & & $13.0 / 13$ \\
$v{ }_{\mu} p \rightarrow v{ }_{\mu} p$ & $0.221 \pm 0.021 \pm[0.003]$ & $0.976 \pm 0.056 \pm[0.002]$ & 0.12 & $3.9 / 10$ \\
$(-){ }_{\mu} e \rightarrow v{ }_{\mu} e$ & $0.228 \pm 0.008 \pm[0.003]$ & $1.015 \pm 0.026 \pm[0.004]$ & 0.19 & $1.6 / 2$ \\
$W, Z$ & $0.229 \pm 0.0064$ & $0.998 \pm 0.0086$ & 0.63 & $145 / 183$ \\
All data & & & & \\
\hline \hline
\end{tabular}

$$
\sin ^{2} \theta^{0} \equiv \sin ^{2} \theta_{W}-\Delta s^{2}
$$

where $\sin ^{2} \theta^{0}$ is the value $(0.242 \pm 0.006)$ obtained for the weak angle from deep-inelastic scattering if all radiative corrections (to both $\sigma^{\mathrm{NC}}$ and $\sigma^{\mathrm{CC}}$ ) are ignored, and $\Delta s^{2}$ represents the radiative corrections. For our canonical $m_{t}$ and $M_{H}, \Delta s^{2}$ is $-0.009 \pm 0.001$. Then
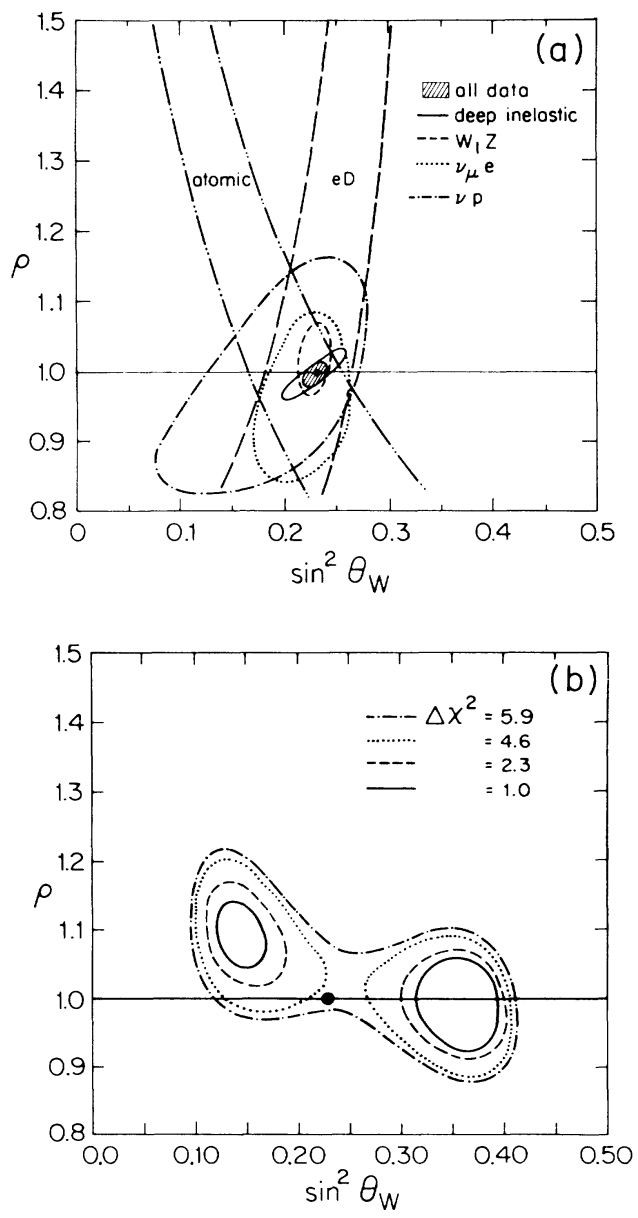

FIG. 3. (a) The allowed regions in the $\sin ^{2} \theta_{W}-\rho$ plane at $90 \%$ C.L. for various individual reactions. (b) The allowed regions for $e^{+} e^{-}$data at $\Delta \chi^{2}=5.9$ (95\% C.L.), 4.6 (90\% C.L.), 2.3 (68\% C.L.), and 1.0 (39\% C.L.), where the $\Delta \chi^{2}$ values are with respect to the minimum at $\sin ^{2} \theta_{W} \simeq 0.14$.

$$
\begin{aligned}
& M_{W}=\frac{A_{0}}{\sin \theta^{0}\left(1-\delta_{W}\right)^{1 / 2}}, \\
& M_{Z}=\frac{A_{0}}{\sin \theta^{0} \cos \theta^{0}\left(1-\delta_{Z}\right)^{1 / 2}},
\end{aligned}
$$

where

$$
\begin{aligned}
\delta_{W} & =\Delta r-\frac{\Delta s^{2}(1-\Delta r)}{\sin ^{2} \theta^{0}} \\
& =\Delta r+0.035 \pm 0.004 \\
\delta_{Z} & =\Delta r-\frac{\Delta s^{2}(1-\Delta r)}{\sin ^{2} \theta^{0}}\left(1-\tan ^{2} \theta^{0}-\Delta s^{2} / \cos ^{2} \theta^{0}\right) \\
& =\Delta r+0.024 \pm 0.003,
\end{aligned}
$$

$\delta_{W}$ represents all of the radiative corrections relating deep-inelastic scattering, the $W$ and $Z$ masses, and muon decay. The allowed region in $\delta_{W}-\sin ^{2} \theta^{0}$ can be obtained from the $\Delta r-\sin ^{2} \theta_{W}$ plot in Fig. 4 by appropriately shifting the horizontal and vertical axes (see the right-hand and top axes in the figure). From (19), the experimental value of $\delta_{W}$ is $0.112 \pm 0.037$. This is consistent with the prediction $\delta_{W}=0.106 \pm 0.004$ for our canonical $m_{t}, M_{H}$

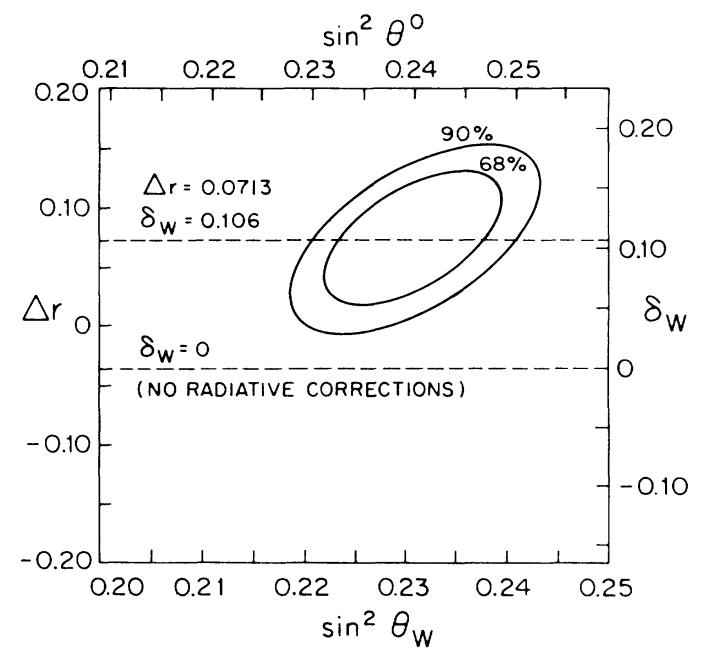

FIG. 4. The allowed region in the $\sin ^{2} \theta_{W}-\Delta r$ (or $\sin ^{2} \theta^{0}-\delta_{W}$ ) plane determined from deep-inelastic (isoscalar) data and the $W$ and $Z$ masses. 


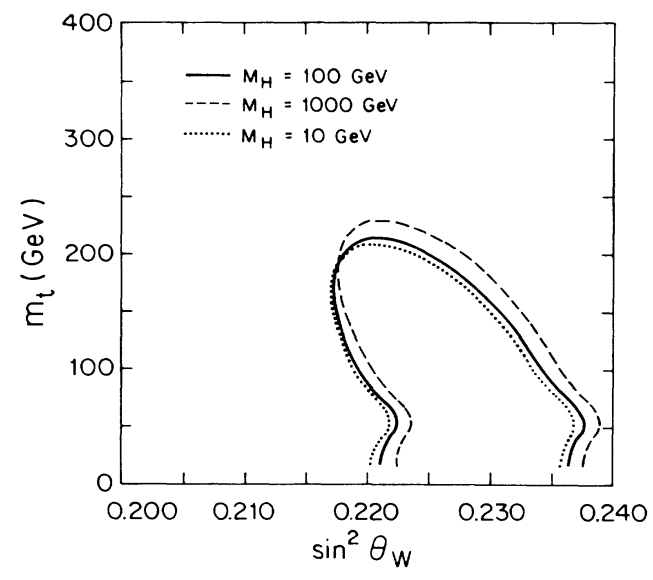

FIG. 5. Allowed regions $(90 \%$ C.L. $)$ in $\sin ^{2} \theta_{W}-m_{t}$ for Higgs-boson masses of 10, 100, and $1000 \mathrm{GeV}$.

assumptions and establishes the existence of electroweak radiative corrections at the $3 \sigma$ level.

A similar conclusion can also be reached from the data in the last two rows of Table VII. The standardmodel predictions for $M_{W}$ and $M_{Z}$ (using $\sin ^{2} \theta_{W}$ from deep-inelastic scattering) are in excellent agreement with observations, but the predictions without radiative corrections [(13) and (17) with $\left.\Delta r=\Delta s^{2}=0\right]$ strongly disagree with experiment.

\section{Heavy fermions}

Within the standard model with three fermion families the only major uncertainties are $m_{t}$ and $M_{H}$, which affect the radiative correction parameters $\Delta r, \rho^{\mathrm{NC}}$, and $\kappa$ in Sec. II. The Higgs-boson mass dependence is small although not completely negligible as long as $M_{H}<1$ $\mathrm{TeV}$. It typically introduces an uncertainity of 0.002 in the $\sin ^{2} \theta_{W}$ value extracted from most reactions other than deep-inelastic scattering. For larger $M_{H}$ the perturbative calculation of radiative corrections becomes suspect (because of the large Higgs-boson self-coupling) so we will restrict ourselves to the case $M_{H}<1 \mathrm{TeV}$.

The sensitivity to $m_{t}$ (or to splittings between fourth family fermions) is much larger. For example, $\Delta r \rightarrow 0$ for $m_{t} \sim 245 \mathrm{GeV}$ and the various $\rho^{\mathrm{NC}}$ and $\kappa$ parameters exhibit similar sensitivity. The $\sin ^{2} \theta_{W}$ values obtained for various reactions are shown in Fig. 1 (and also in Table XVI) for $m_{t}=45,100,200$, and $400 \mathrm{GeV}$. There is little sensitivity for $m_{t} \leq 100 \mathrm{GeV}$. For larger values the $\sin ^{2} \theta_{W}$ from deep-inelastic scattering stays almost constant (due to a near cancellation of the variations due to $\rho_{v N}^{\mathrm{NC}}$ and $\left.\kappa_{v N}\right)$, while the other determinations decrease. Clearly, the data are inconsistent with $m_{t}$ much larger than $200 \mathrm{GeV}$. To quantify this we have performed fits to $m_{t}$ and $\sin ^{2} \theta_{W}$ for various (fixed) values of $M_{H}$, including the full $m_{t}$ and $M_{H}$ dependence of the radiative corrections to all processes. The allowed regions for $M_{H}=10,100$, and $1000 \mathrm{GeV}$ are shown in Fig. 5. The corresponding upper limits on $m_{t}$ alone ${ }^{108}$ are
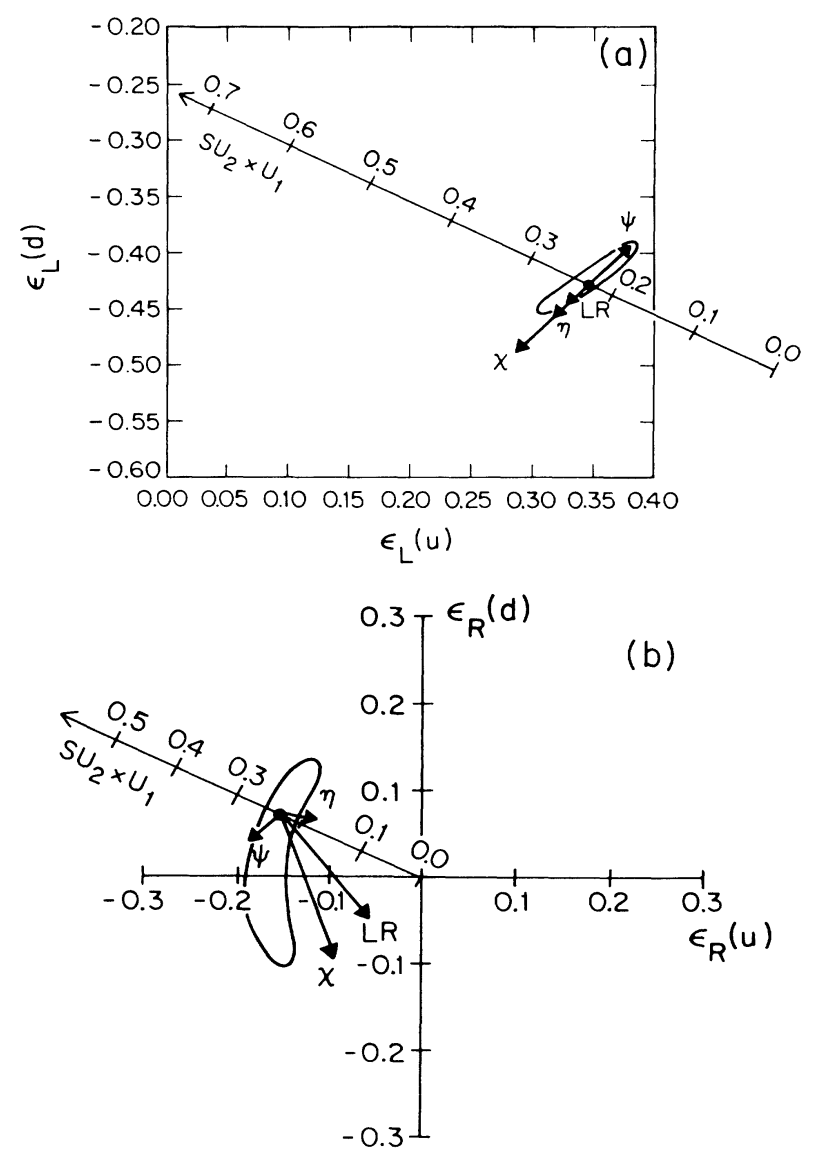

FIG. 6. (a) Allowed regions $(90 \%$ C.L.) for the modelindependent $v q$ parameters $\epsilon_{L}(u)-\epsilon_{L}(d)$, (b) allowed regions for $\epsilon_{R}(u)-\epsilon_{R}(d)$. Also shown are the predictions of the standard model as a function of $\sin ^{2} \theta_{W}$ and the changes that would be induced (relative to the standard model) for various additional $Z$ bosons with $M_{Z_{2}}=M_{Z_{1}}$, and $\theta=0$.

$m_{t}<175,180$, and $200 \mathrm{GeV}$ for $M_{H}=10,100$, and 1000 $\mathrm{GeV}$, respectively, all at $90 \%$ C.L. Similar limits can be obtained from the $\Delta r$ - $\sin ^{2} \theta_{W}$ fits, which use only deepinelastic scattering and the $W$ and $Z$ masses. It should be emphasized that all of these limits assume that there is no new physics that produces compensating effects.

One can place similar limits on the mass splittings between the quarks or leptons in a fourth fermion family: $\left|m_{t^{\prime}}-m_{b^{\prime}}\right|<180 \mathrm{GeV}$ and $\left|m_{L}-m_{v_{L}}\right|<310 \mathrm{GeV}$ for $M_{H}=100 \mathrm{GeV}$. Such splittings give positive contributions to $\rho, \kappa$, and $-\Delta r$, independent of the sign of $m_{t^{\prime}}-m_{b^{\prime}}$, so they cannot cancel the effect of large $m_{t}$ (Ref. 109). (This is true for most but not all possible extensions of the standard model. ${ }^{110}$ ) These limits become more stringent as the average doublet mass increases.

\section{E. Model-independent fits}

Model-independent fits to the $v q, v e$, and $e q$ are presented in Figs. 6-8 and Table XV. The $v q$ chiral cou- 
plings $\epsilon_{i}(u), \epsilon_{i}(d), i=L, R$ are uniquely determined (Fig. 6) by the data in Tables I-III and are in good agreement with the standard-model predictions.

The ve parameters $g_{A}^{e}, g_{V}^{e}$ are shown in Fig. 7. The $\left(v_{\mu} e\right.$ data alone allow four solutions (which differ by $g_{i}^{e} \rightarrow-g_{i}^{e}$ and by $\left.g_{V}^{e} \leftrightarrow g_{A}^{e}\right)$. The reactor $\bar{v}_{e} e$ results eliminate solution C (Ref. 111), while the Los Alamos $v_{e} e$ experiment eliminates solutions $C$ and $D$. The remaining two solutions [axial-vector-dominant (A) and vectordominant (B)] are consistent with all data. ${ }^{112}$ The axialvector-dominant solution agrees with the standard model and is shown in Fig. 7(b).

Assuming a single $Z$ boson, the $e^{+} e^{-}$data (mainly the forward-backward asymmetries) are very useful for testing $e-\mu-\tau$ universality in the axial-vector couplings (the vector couplings are consistent with zero and are not well determined). Assuming $\rho=1$ and the canonical
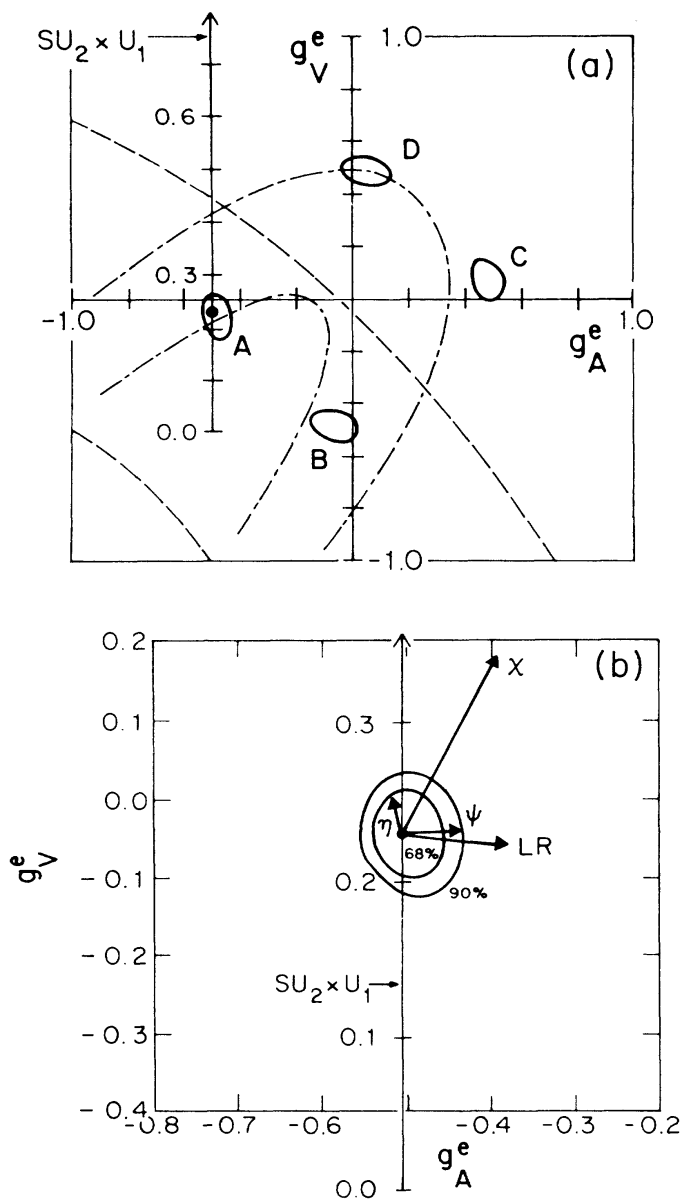

FIG. 7. (a) Allowed regions (90\% C.L.) for the ve parameters $g_{V}^{e}-g_{A}^{e}$ for various reactions: ${ }^{(}{ }_{\mu}^{\prime} e$ (solid lines), reactor $\bar{v}_{e} e$ (dot-dashed line), and $v_{e} e$ (dashed line). (b) Allowed (axialvector-dominant) region (A) $(90 \%$ and $68 \%$ C.L.) from the global fit to all ve data [the second, vector-dominant solution (B) is off scale]. Also shown are the standard-model predictions as a function of $\sin ^{2} \theta_{W}$ and changes that would be induced by additional $Z$ bosons with $M_{Z_{2}}=M_{Z_{1}}$ and $\theta=0$.
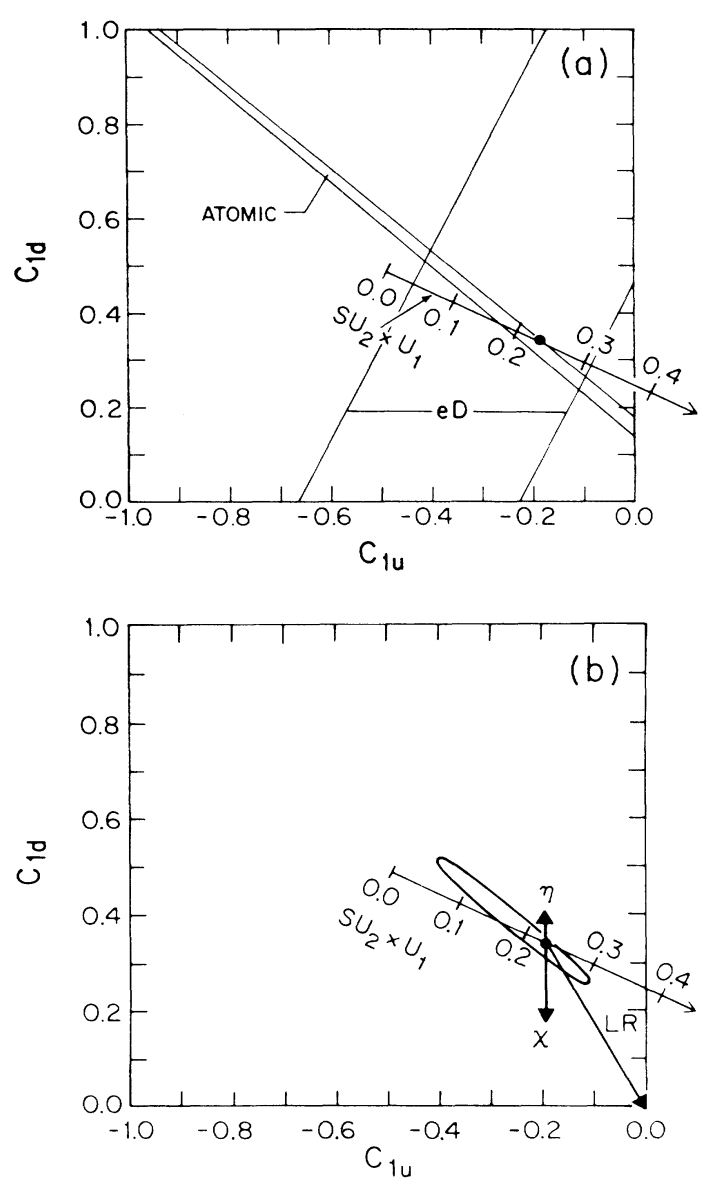

FIG. 8. (a) Allowed regions (90\% C.L.) in $C_{1 u}-C_{1 d}$ from the SLAC $e \mathrm{D}$ experiment and atomic parity violation. (b) Combined fit. Also shown are the predictions of the standard model as a function of $\sin ^{2} \theta_{W}$ and the changes that would be induced by extra $Z$ bosons with $M_{Z_{2}}=M_{Z_{1}}$ and $\theta=0$. (The $Z_{\psi}$ does not contribute to $C_{1 i}$.)

expression for $h_{V V}$, a fit to all data yields $h_{A A}^{\mu}=g_{A}^{e} g_{A}^{\mu}=0.272 \pm 0.015$ and $h_{A A}^{\tau}=g_{A}^{e} g_{A}^{\tau}=0.232$ \pm 0.026 , very close to the standard-model prediction $\frac{1}{4}$. For $g_{A}^{e}=-\frac{1}{2}$, these imply $g_{A}^{\mu}=-0.54 \pm 0.03$ and $g_{A}^{\tau}=-0.46 \pm 0.05$, in impressive agreement with universality.

The allowed region for the $e q$ couplings $C_{1 u}$ and $C_{1 d}$ are shown in Fig. 8. These are obtained from a simultaneous fit of $C_{1 u}, C_{1 d}, C_{2 u}$, and $C_{2 d}$ to the SLAC and atomic parity-violation data. (The $\mu \mathrm{C}$ asymmetries are not used because they depend on parity-conserving couplings as well.) The agreement of $C_{1 u}$ and $C_{1 d}$ with the standard-model prediction is impressive. $C_{2 u}$ and $C_{2 d}$ are only weakly constrained and are not displayed. ${ }^{113}$

\section{F. Additional $Z$ bosons (Refs. 114-116)}

Many extensions of the standard-model predict the existence of additional $Z$ bosons. For the simplest case (one extra $Z$ ) the physical (mass eigenstate) bosons are 
TABLE XV. Values of the model-independent neutral-current parameters, compared with the standard-model prediction for $\sin ^{2} \theta_{W}=0.230$. We do not give correlations for the neutrino hadron couplings because of the non-Gaussian $\chi^{2}$ distributions. However, the neutrino hadron constraints are accurately represented by the ranges listed for the variables $g_{i}{ }^{2} \equiv \epsilon_{i}(u)^{2}+\epsilon_{i}(d)^{2}$ and

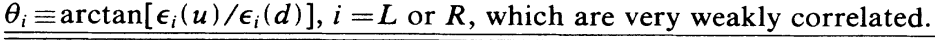

\begin{tabular}{lcccc}
\hline \hline Quantity & $\begin{array}{c}\text { Experimental } \\
\text { value }\end{array}$ & $\begin{array}{c}\text { Standard-model } \\
\text { prediction }\end{array}$ & Correlations & $\chi^{2} / \mathrm{DF}$ \\
\hline$\epsilon_{L}(u)$ & $0.339 \pm 0.017$ & 0.345 & & $33.6 / 48$ \\
$\epsilon_{L}(d)$ & $-0.429 \pm 0.014$ & -0.427 & & \\
$\epsilon_{R}(u)$ & $-0.172 \pm 0.014$ & -0.152 & & \\
$\epsilon_{R}(d)$ & $-0.011_{-0.057}^{+0.081}$ & 0.076 & & \\
$g_{L}{ }^{2}$ & $0.2996 \pm 0.0044$ & 0.301 & & $33.6 / 48$ \\
$g_{R}{ }^{2}$ & $0.0298 \pm 0.0038$ & 0.029 & & \\
$\theta_{L}$ & $2.47 \pm 0.04$ & 2.46 & & \\
$\theta_{R}$ & $4.65_{-0.32}^{+0.48}$ & 5.18 & & \\
$g_{A}^{e}$ & $-0.498 \pm 0.027$ & -0.503 & & \\
$g_{V}^{e}$ & $-0.044 \pm 0.036$ & -0.045 & & \\
$C_{1 u}$ & $-0.249 \pm 0.071$ & -0.191 & & \\
$C_{1 d}$ & $0.381 \pm 0.064$ & 0.340 & -0.98 & \\
$C_{2 u}-\frac{1}{2} C_{2 d}$ & $0.19 \pm 0.37$ & -0.039 & & \\
\hline \hline
\end{tabular}

$$
\begin{aligned}
& Z_{1}=Z_{1}^{0} \cos \theta+Z_{2}^{0} \sin \theta, \\
& Z_{2}=-Z_{1}^{0} \sin \theta+Z_{2}^{0} \cos \theta,
\end{aligned}
$$

where, under reasonable assumptions, the lighter boson $Z_{1}$ is the particle observed by UA1 and UA2, $Z_{1}^{0}$ is the $\mathrm{SU}(2) \times \mathrm{U}(1)$ boson which couples to $g_{1}\left(J_{3 L}\right.$ $\left.-\sin ^{2} \theta_{W} J_{\mathrm{EM}}\right), Z_{2}^{0}$ couples to a new current $g_{2} J_{2}^{0}$, and $\theta$ is a mixing angle. The extra $Z$ manifests itself (a) because the $Z_{1}$ mass is reduced relative to (13) by mixing, (b) because the $Z_{1}$ couplings are modified by mixing, and (c) by $Z_{2}$ exchange. The changes in the standard-model predictions for $v q$, $v e$, and $e q$ couplings due to the exchange of various $Z_{2}$ 's with $\theta=0$ and $M_{Z_{2}}=M_{Z_{1}}$, are indicated by arrows in Figs. 6-8. (The labels $L R, \chi, \psi$, and $\eta$ refer to the theoretical models to be discussed below.) For heavier $Z_{2}$ the changes scale as $\left(M_{Z_{1}} / M_{Z_{2}}\right)^{2}$.

It is not practical to consider a totally arbitrary $g_{2} J_{2}^{0}$, so we focus on several theoretically interesting special cases.

(i) $Z_{L R}$, which occurs in $\mathrm{SU}(2)_{L} \times \mathrm{SU}(2)_{R} \times \mathrm{U}(1)$ models. One has

$$
J_{2}^{0}=J_{L R}=\left(\frac{3}{5}\right)^{1 / 2}\left[\alpha J_{3 R}-\frac{1}{2 \alpha} J_{B-L}\right],
$$

where

$$
\alpha=\left[\left(\frac{g_{R}}{g_{L}}\right)^{2}\left[\frac{1-\sin ^{2} \theta_{W}}{\sin ^{2} \theta_{W}}\right)-1\right]^{1 / 2}
$$

and $\left(g_{2} / g_{1}\right)^{2}=5 \sin ^{2} \theta_{W} / 3$. In most versions of the model the extra charged $W$ must be more massive than several TeV (Ref. 117), but the limits on $M_{Z_{2}}$ are much weaker.

(ii) $Z_{\chi}$, which occurs in $\mathrm{SO}(10)$ models breaking to $\mathrm{SU}(5) \times \mathrm{U}(1)_{\chi}$. The $\chi$ charge is $Q_{\chi}=-1 / 2 \sqrt{10}$ for $\left(u, d, \bar{u}, e^{+}\right)_{L}$ and $Q_{\chi}=3 / 2 \sqrt{10}$ for $\left(\bar{d}, v, e^{-}\right)_{L}$, and $\left(g_{2} / g_{1}\right)^{2}=5 \lambda_{\chi} \sin ^{2} \theta_{W} / 3$, where $\lambda_{\chi} \sim\left(\frac{2}{3}-1\right)$ for reasonable $\mathrm{SO}(10)$-breaking chains. ${ }^{118}$

(iii) $Z_{\psi}$, which occurs in $\mathrm{E}_{6}$ models breaking to $\mathrm{SO}(10) \times \mathrm{U}(1)_{\psi}$. The $Q_{\psi}$ charge is $1 / \sqrt{24}$ for $\left(u, \bar{u}, d, \bar{d}, v, e^{-}, e^{+}\right)_{L}$ and $\left(g_{2} / g_{1}\right)^{2}=5 \lambda_{\psi} \sin ^{2} \theta_{W} / 3$ with $\lambda_{\psi} \leq \lambda_{\chi} \leq 1$.

(iv) $Z_{\eta}$, which occurs in the breaking $\mathrm{E}_{6} \rightarrow \mathrm{SU}(3)$ $\times \mathrm{SU}(2) \times \mathrm{U}(1) \times \mathrm{U}(1)_{\eta}$ expected in some superstring models. One has $Q_{\eta}=\sqrt{3 / 8} Q_{\chi}-\sqrt{5 / 8} Q_{\psi} \quad$ and $\left(g_{2} / g_{1}\right)^{2}=5 \lambda_{\eta} \sin ^{2} \theta_{W} / 3$.

(v) $\quad Z(\beta)=\cos \beta Z_{\chi}+\sin \beta Z_{\psi}$, with $\left(g_{2} / g_{1}\right)^{2}$ $=5 \lambda_{\beta} \sin ^{2} \theta_{W} / 3$. This is the extra boson in $\mathrm{E}_{6}$ models which break to $G \times \mathrm{U}(1)_{\beta}$, where $G$ contains the standard model. The $Z_{\chi}, Z_{\psi}$, and $Z_{\eta}$ are special cases with $\beta=0, \pi / 2$ and $-Z_{\eta}=Z(\beta=\pi-\arctan \sqrt{5 / 3})$, respectively.

We will follow the formalism in Ref. 115. We assume $\lambda_{\chi}=\lambda_{\psi}=\lambda_{\eta}=\lambda_{\beta}=1$, which occurs if the underlying group breaks directly to $\mathrm{SU}(3) \times \mathrm{SU}(2) \times \mathrm{U}(1) \times \mathrm{U}^{\prime}(1)$ (limits on $M_{Z_{2}}$ and $\theta$ scale roughly as $\sqrt{\lambda}$ and $1 / \sqrt{\lambda}$, respectively). Limits on $M_{Z_{2}}$ and $\theta$ are presented for two cases.

(a) The constrained Higgs case. This case, which is the analog of $\rho=1$ in $\mathrm{SU}(2) \times \mathrm{U}(1)$, occurs if all $\mathrm{SU}(2) \times \mathbf{U}(1)$ breaking is due to Higgs doublets (this is expected in superstring models). The free parameters are $\sin ^{2} \theta_{W}, M_{Z_{2}}$, and $\theta . M_{Z_{1}}$ is related by

$$
\tan ^{2} \theta=\frac{M_{0}{ }^{2}-M_{Z_{1}}{ }^{2}}{M_{Z_{2}}{ }^{2}-M_{0}{ }^{2}},
$$

where $M_{0}=M_{W} / \cos \theta_{W}$ would be the $Z_{1}^{0}$ mass in the absence of mixing.

(b) The unconstrained Higgs-boson case. This is the analog of $\rho \neq 1$ and occurs if there are Higgs triplets, 

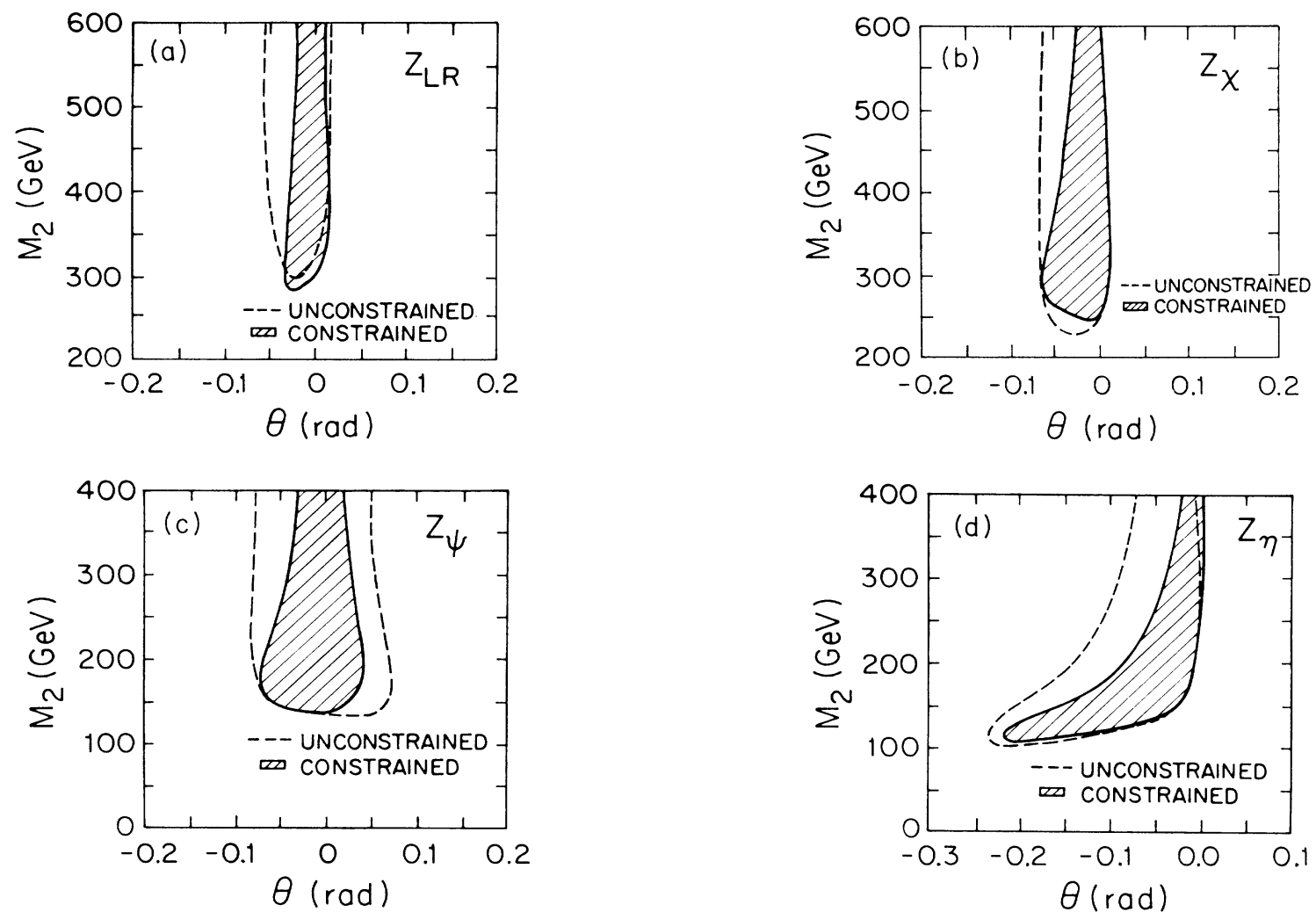

FIG. 9. Allowed regions (90\% C.L.) in $M_{Z_{2}}$ and $\theta$ for (a) $Z_{L R}$, (b) $Z_{\chi}$, (c) $Z_{\psi}$, (d) $Z_{\eta}$. (Note the shifted $\theta$ scale for $Z_{n}$.) Limits are shown for both constrained and unconstrained Higgs sectors. The 90\%-C.L. lower limits on the masses alone, for the constrained and unconstrained cases, respectively, are 325 and $343 \mathrm{GeV}\left(\boldsymbol{Z}_{L R}\right), 273$ and $249 \mathrm{GeV}\left(\boldsymbol{Z}_{\chi}\right), 154$ and $151 \mathrm{GeV}\left(\boldsymbol{Z}_{\psi}\right)$, and 111 and $112 \mathrm{GeV}\left(Z_{\eta}\right)$.

etc., with significant VEV's. In this case $\sin ^{2} \theta_{W}, M_{Z_{1}}$, $M_{Z_{2}}$, and $\theta$ are all arbitrary.

The allowed regions of $M_{Z_{2}}$ vs $\theta$ are shown for the $Z_{L R}, Z_{\chi}, Z_{\psi}$, and $Z_{\eta}$ in Fig. 9. Except for $Z_{\eta}$, the limits show a modest but not dramatic improvement over those in Ref. 115 (which was based on a much earlier data set). The $90 \%$-C.L. lower limits on $M_{Z_{2}}$ and $\theta$ range are shown for $Z(\beta)$ as a function of $\cos \beta$ in Fig. 10. $|\theta|$ must be smaller than $\simeq 0.05$ except for a small region near the $Z_{\eta}$. The relative weakness of the constraints on $Z_{2}$ is due to the fact that most theoretically favored extra $Z$ 's tend to couple fairly weakly to the ordinary quarks and leptons (and more strongly to exotic fermions such as heavy Majorana neutrinos ${ }^{119}$ ). The weakest limits are in the range $-0.8<\cos \beta<0$, for which the $Z_{2}^{0}$ coupling to neutrinos is especially small. (The actual $Z_{2}^{0} v v$ decoupling occurs for $\cos \beta \simeq-0.4$. )

The $Z_{\eta}$ limits in Figs. 9(d) and 10 deserve special comment. There is a contour of $M_{Z_{2}}-\theta$ values extending through the middle of the allowed region in Fig. 9(d) for which the $v q$ and $v e$ couplings as well as $C_{1 i}$ and $h_{A A}$ are the same as in the standard model, due to a cancellation between the effects of mixing, $\Delta M_{Z_{1}}$, and $Z_{2}$ ex-
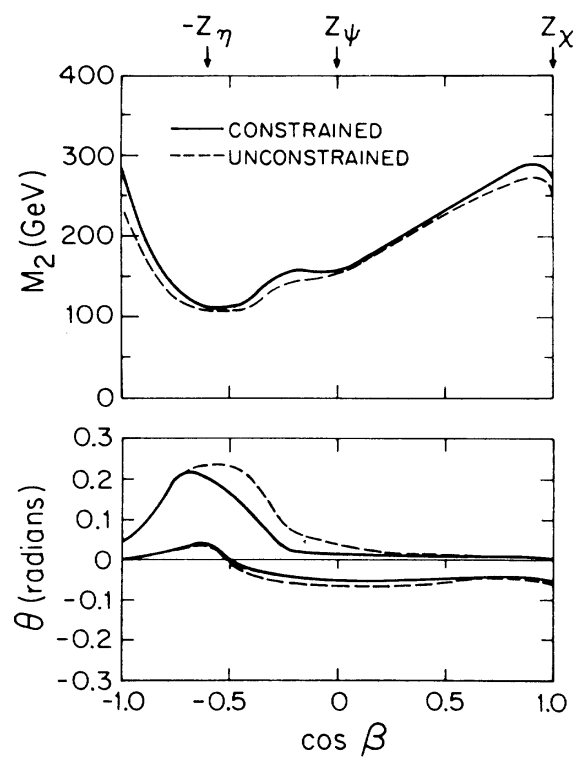

FIG. 10. Lower limits on $M_{Z_{2}}$ and allowed $\theta$ range (both at 90\% C.L.) for an $E_{6}$ boson $Z(\beta)=\cos \beta Z_{\chi}+\sin \beta Z_{\psi}$ for constrained and unconstrained Higgs bosons. The special cases $Z_{\chi}, Z_{\psi}$, and $-Z_{\eta}$ are indicated. 
change. That accounts for the very weak limits on $M_{Z_{2}}$ (especially for the unconstrained case, for which even $\boldsymbol{M}_{Z_{1}}$ is unimportant) and the asymmetry in the sign of $\theta$. In fact, it is apparent from Figs. 9(d) and 10 that the data prefer a low mass $\left(M_{Z_{2}} \sim 130 \mathrm{GeV}\right) Z_{\eta}$ over the standard model $\left(M_{Z_{2}}=\infty\right)$. This is because there are no cancellations in the $e^{+} e^{-}$vector coupling $h_{V V}$; the slight energy dependence of $R_{l l}$ favors a low mass $Z_{\eta}$, which has a significant vector coupling to electrons. Comments similar to those in Secs. IV A and IV B concerning possible energy-dependent systematic effects and the statistical significance of the result apply here. In particular, the standard model is $\Delta \chi^{2} \sim 5.2$ above the minimum for the constrained fit and falls within the 93\%-C.L. contour, so there is no compelling evidence for new physics.

At the present time the indirect limits on heavy $Z$ 's (with $\lambda \sim 1$ ) from the neutral current ${ }^{120}$ are somewhat more stringent ${ }^{115,116}$ than limits from direct searches $\bar{p} p \rightarrow Z_{2}+X, Z_{2} \rightarrow l^{+} l^{-}$at the CERN S $\bar{p} p$ S except for a small region in $\beta$ near the $Z_{\eta}$. This situation will presumably change in the near future: for example, the Fermilab $\bar{p} p$ collider should be sensitive to bosons of the type described here up to around $400 \mathrm{GeV}$ and the Superconducting Super Collider (SSC) would be sensitive up to several TeV.

\section{GRAND UNIFICATION}

For discussions involving grand unified theories (GUT's), it is convenient to define a running $\sin ^{2} \hat{\theta}_{W}(\mu)$ by $\overline{\mathrm{MS}}$ (modified minimal subtraction ${ }^{121}$ ) where the variable mass scale $(\mu)$ is conventionally chosen to be $M_{W}$ for electroweak processes. ${ }^{122}$ That definition is quite insensitive to the Higgs-scalar and top-quark masses. It is also straightforward to predict $\sin ^{2} \hat{\theta}_{W}\left(M_{W}\right)$ in GUT's.

The relationship between $\sin ^{2} \theta_{W} \equiv 1-M_{W}{ }^{2} / M_{Z}{ }^{2}$ and $\sin ^{2} \hat{\theta}_{W}\left(M_{W}\right)$ is given by ${ }^{14}$ (for $M_{H}=100 \mathrm{GeV}$ )

$\sin ^{2} \hat{\theta}_{W}\left(M_{W}\right)=\left[0.9945-G\left(m_{t}{ }^{2} / M_{W}{ }^{2}\right)\right] \sin ^{2} \theta_{W}$,

where $G\left(m_{t}{ }^{2} / M_{W}{ }^{2}\right)$ is a complicated function ${ }^{17}$ with $G(0)=0$. For $m_{t}=45 \mathrm{GeV}, G(0.31)=0.0038$; thus $\sin ^{2} \hat{\theta}_{W}\left(M_{W}\right)=0.9907 \sin ^{2} \theta_{W}$, implying the worldaverage value ${ }^{123}$

$$
\sin ^{2} \hat{\theta}_{W}\left(M_{W}\right)=0.228 \pm 0.0044 .
$$

For other $m_{t}$ values, the relationship between $\sin ^{2} \theta_{W}$ and $\sin ^{2} \hat{\theta}_{W}\left(M_{W}\right)$ is illustrated in Table XVI. We note that if one extracts $\sin ^{2} \hat{\theta}_{W}\left(M_{W}\right)$ directly from experiment, only the deep-inelastic neutrino scattering value is very sensitive to $m_{t}$ - just the opposite of $\sin ^{2} \theta_{W}$.

Employing $\alpha^{-1}\left(M_{W}\right)=127.8 \pm 0.3$, one finds, for the $\mathrm{SU}(2)_{L} \times \mathrm{U}(1) \overline{\mathrm{MS}}$ couplings,

$$
\begin{aligned}
& \alpha_{1}\left(M_{W}\right)=\frac{5 \alpha\left(M_{W}\right)}{3 \cos ^{2} \hat{\theta}_{W}\left(M_{W}\right)}=0.0169 \pm 0.0001, \\
& \alpha_{2}\left(M_{W}\right)=\frac{\alpha\left(M_{W}\right)}{\sin ^{2} \hat{\theta}_{W}\left(M_{W}\right)}=0.0344 \pm 0.0007 .
\end{aligned}
$$

Note that they are now very well determined. For comparison, the $\mathrm{SU}(3)_{c} \overline{\mathrm{MS}}$ coupling is given by

$$
\alpha_{3}\left(M_{W}\right)=0.107_{-0.008}^{+0.013}
$$

where $\Lambda \frac{(4)}{\mathrm{MS}}=150_{-75}^{+150} \mathrm{MeV}$ is used. Evolving the gauge couplings to higher energies using the three-generation $\beta$ functions ${ }^{122}$

$$
\begin{aligned}
& \mu \frac{\partial}{\partial \mu} \alpha_{i}(\mu)=b_{i} \alpha_{i}{ }^{2}+\cdots, \quad i=1,2,3 \\
& \left(\begin{array}{l}
b_{1} \\
b_{2} \\
b_{3}
\end{array}\right)=\frac{-1}{2 \pi}\left(\begin{array}{c}
-\frac{41}{10} \\
\frac{19}{6} \\
7
\end{array}\right),
\end{aligned}
$$

which assumes no new physics beyond the standard model, gives the curves in Fig. 11. It is apparent that the couplings are running towards one another, thus strongly suggesting unification, but they do not meet at a point. [In past years, a lower $\sin ^{2} \hat{\theta}_{W}\left(M_{W}\right) \simeq 0.214$ led to unification at about $\mu=2 \times 10^{14} \mathrm{GeV}$.] To achieve unification requires some new physics threshold before $10^{15} \mathrm{GeV}$ which modifies the evolution equations in Eq. (28). The issue is the following: What new physics and at what new energy is it manifested?

The above remarks are nicely illustrated by the minimal SU(5) model ${ }^{124}$ with a "great desert," i.e., no new physics between $M_{W}$ and the unification mass scale $\boldsymbol{M}_{X}$. Using $\alpha^{-1}\left(\boldsymbol{M}_{W}\right)=127.8 \pm 0.3$ and $\alpha_{3}\left(M_{W}\right)$ given in (27) that model predicts

$$
\begin{aligned}
& \sin ^{2} \hat{\theta}_{W}\left(M_{W}\right)=0.214_{-0.004}^{+0.003}, \\
& M_{X}=2.0_{-1.0}^{+2.1} \times 10^{14} \mathrm{GeV},
\end{aligned}
$$

essentially independent of $m_{t}$ and $M_{H}$. The $\sin ^{2} \hat{\theta}_{W}\left(M_{W}\right)$ prediction disagrees with the world average in (25) by about 2.5 standard deviations (adding errors in quadrature) and thereby points out the need for new physics in

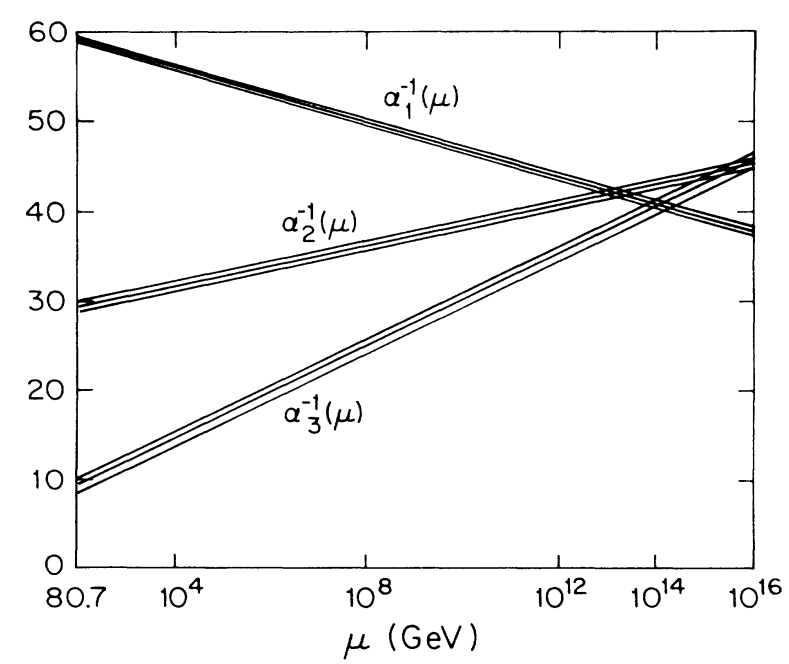

FIG. 11. Evolution of the couplings $\alpha_{i}{ }^{-1}(\mu), i=1,2,3$ assuming no new physics beyond the standard model. 
TABLE XVI. Values of $\sin ^{2} \theta_{W}$ determined by a global fit to all data for various $m_{t}$ and $M_{H}$, the ratio $\sin ^{2} \hat{\theta}_{W}\left(M_{W}\right) / \sin ^{2} \theta_{W}$, and the corresponding value of $\sin ^{2} \hat{\theta}_{W}\left(M_{W}\right)$. The latter quantity should be compared with the SU(5) prediction $0.214_{-0.004}^{+0.003}$ and the SUSY GUT prediction in (31).

\begin{tabular}{cclcl}
\hline \hline$m_{t}(\mathrm{GeV})$ & $M_{H}(\mathrm{GeV})$ & \multicolumn{1}{c}{$\sin ^{2} \theta_{W}$} & $\frac{\sin ^{2} \hat{\theta}_{W}\left(\boldsymbol{M}_{W}\right)}{\sin ^{2} \theta_{W}}$ & $\sin ^{2} \hat{\theta}_{W}\left(M_{W}\right)$ \\
\hline 25 & 100 & $0.229 \pm 0.004$ & 0.994 & $0.227 \pm 0.004$ \\
45 & 100 & 0.230 & 0.991 & 0.228 \\
60 & 100 & 0.230 & 0.991 & 0.228 \\
100 & 100 & 0.227 & 1.01 & 0.229 \\
200 & 100 & 0.222 & 1.05 & 0.233 \\
400 & 100 & 0.209 & 1.18 & $0.248 \pm 0.005$ \\
45 & 10 & 0.229 & 0.994 & 0.228 \\
45 & 1000 & 0.231 & 0.982 & 0.227 \\
\hline \hline
\end{tabular}

the desert. As is apparent in Table XVI the discrepancy is made worse for large $m_{t}$ : although the experimental value of $\sin ^{2} \theta_{W}$ decreases with $m_{t}$ the $\sin ^{2} \hat{\theta}_{W}$ value actually increases. Of course, proton decay lifetime bounds $^{125}$ have required $M_{X}>7 \times 10^{14} \mathrm{GeV}$ for some time and were the first indication of a problem with minimal SU(5). $\sin ^{2} \hat{\theta}_{W}\left(M_{W}\right)$ confirms and strengthens the arguments against a "great desert" scenario.

A nice example of how new physics thresholds can bring GUT's into better accord with low-energy phenomenology is provided by supersymmetry (SUSY). In SUSY GUT's, the evolution equations in (28) are modified when one evolves past the scale of supersymmetry breaking $M_{\text {SUSY }}$ such that, for two light Higgs doublets, ${ }^{126}$

$$
\left(\begin{array}{l}
b_{1} \\
b_{2} \\
b_{3}
\end{array}\right)=\frac{-1}{2 \pi}\left(\begin{array}{c}
-\frac{33}{5} \\
-1 \\
3
\end{array}\right) \text {. }
$$

Then, using $\alpha^{-1}\left(M_{W}\right)$ and $\alpha_{3}\left(M_{W}\right)$ as input, one finds the prediction

$\sin ^{2} \hat{\theta}_{W}\left(M_{W}\right)=0.237_{-0.004}^{+0.003}-\frac{4}{15} \frac{\alpha\left(M_{W}\right)}{\pi} \ln \left(\frac{M_{\text {SUSY }}}{M_{W}}\right)$.

That prediction appears to be closer to the value $0.228 \pm 0.0044$ suggested by experiment, but somewhat high unless $\Lambda \frac{(4)}{\mathrm{MS}}$ is on the high side $(\simeq 300 \mathrm{MeV}), M_{\text {SUSY }}$ is larger than the usually assumed range $100-1000 \mathrm{GeV}$, or $m_{t}$ is large $(\simeq 200 \mathrm{GeV})$. (In addition, $M_{X}$ is predicted to be $\simeq 5 \times 10^{15} \mathrm{GeV}$, which is consistent with proton decay bounds.) Of course, the assumption of a single new mass scale $M_{\text {SUSY }}$ in SUSY models is too simplistic to take the exact prediction in Eq. (31) too seriously. Properly accounting for mass splittings and allowing for additional Higgs scalars could easily reduce $\sin ^{2} \hat{\theta}_{W}\left(M_{W}\right)$. One may interpret the new higher experimental value of $\sin ^{2} \hat{\theta}_{W}\left(M_{W}\right)$ as being consistent with SUSY GUT's and perhaps even the first harbinger of supersymmetry.

\section{CONCLUSIONS}

The weak neutral current (including the $W$ and $Z$ masses) has long been a major quantitative test of the standard model. A number of recent high-precision experiments allow even more stringent tests of the model and limits on deviations. We have made a careful analysis of existing neutral-current data, attempting to use the best possible theoretical expressions for each reaction, to make realistic estimates of theoretical uncertainties, and to treat similar experiments in a uniform way.

Our major conclusions are as follows. (a) There is no evidence for any deviation from the standard model. (b) A global average to all data yields $\sin ^{2} \theta_{W}=0.230$ \pm 0.0048 . (c) This corresponds to the $\overline{\mathrm{MS}}$ definition $\sin ^{2} \hat{\theta}_{W}\left(M_{W}\right)=0.228 \pm 0.0044$ for $m_{t}$ fixed at $45 \mathrm{GeV}$. This is larger by about $2.5 \sigma$ than the prediction $0.214_{-0.004}^{+0.003}$ of minimal SU(5) and other "great desert" models, and closer to (though still somewhat below) the prediction $(\sim 0.237)$ of many supersymmetric GUT's. This conclusion continues to hold for other values of $m_{t}$ and $M_{H}$. (d) Allowing $\rho \equiv M_{W}{ }^{2} /\left(M_{Z}{ }^{2} \cos ^{2} \theta_{W}\right)$ to vary, one finds $\rho=0.998 \pm 0.0086$, which is impressively close to unity and which places upper limits of 5-8\% on the vacuum expectation values (relative to the ordinary Higgs doublet) of many nonstandard Higgs representations. (e) The radiative correction parameter $\delta_{W}$ is determined to be $0.112 \pm 0.037$, consistent with the expectation 0.106 for $m_{t} \sim 45 \mathrm{GeV}$ and establishing the existence of radiative corrections at the $3 \sigma$ level. (f) The radiative corrections are very sensitive to $m_{t}$ and the mass splittings between additional quarks or leptons. Consistency of the various reactions requires $m_{t}<180$ $\mathrm{GeV}$ (90\% C.L.) for $M_{H}=100 \mathrm{GeV}$, assuming no (compensating) new physics beyond the standard model. (g) Most of the $v q$, $v e, e q$, and $e^{+} e^{-}$neutral-current couplings are now determined uniquely and precisely (Figs. 6-8). (h) At present the weak neutral current (and the $W$ and $Z$ ) give the best limits on the masses and mixings of many theoretically popular additional $Z$ bosons, but the limits (typically $120-300 \mathrm{GeV}$ ) are still relatively weak. 


\section{ACKNOWLEDGMENTS}

It is a pleasure to thank F. Avignone, M. A. Bouchiat, R. Brock, S. Burke, H. Chen, F. Dydak, F. Gilman, I. Hinchliffe, R. W. L. Jones, J. E. Kim, F. Merritt, M. Murtagh, G. Myatt, J. Panman, S. Pakvasa, F. Perrier, P. G. H. Sandars, C. Santoni, J. Smith, P. Souder, F. Taylor, P. Vogel, and C. Wieman for their advice and help. This work was supported in part by Department of Energy Contracts Nos. DE-AC02-76-ER0-3071, DEAC02-76CH00016, and DE-AC02-87ER-40325-TASK B1 and by NSF Grant No. PHY-8116102.

\section{APPENDIX: THE CHARM THRESHOLD}

The major theoretical uncertainty in deep-inelastic scattering from isoscalar targets is the charm threshold, which is mainly important for the charged-current denominators in $R_{v}$ and $R_{\bar{v}}$.

There are several related uncertainties. (a) The elements $U_{c d}$ and $U_{c s}$ of the KMC matrix. For three fermion families these are well determined by unitarity, but for $>3$ families they are poorly known. (b) The moment $S=\int_{0}^{1} x s(x) d x$ of the $s$ sea. (c) The shapes of $s(x), \bar{s}(x)$, and the light-quark distributions. (d) The suppression for $d(x), s(x) \rightarrow c$ or $\bar{d}(x), \bar{s}(x) \rightarrow \bar{c}$. We denote the suppression factors, suitably weighed over $x$ and the target composition, by $\xi_{d}, \xi_{\bar{d}}$, and $\xi_{s} \simeq \xi_{\bar{s}}$.

A major source of information on these quantities is dimuon production $\nu N \rightarrow \mu^{-} \mu^{+} X$, where the extra muon comes from $c(\bar{c})$ decay. From the $v$ and $\bar{v}$ dimuon cross sections one can determine $e^{127,128}$ the quantities

$$
\left|U_{c d}\right|^{2} \xi_{d} B_{\mu}
$$

and

$$
\left|U_{c s}\right|^{2}\left(\frac{2 S}{U+D}\right) \xi_{s} B_{\mu},
$$

where $B_{\mu}$ is the branching ratio for $c \rightarrow \mu$ averaged over the charmed particles produced at the hadronic vertex; however it is difficult to separate the various factors $U_{c d}$, $U_{c s}, S, \xi_{d}$, and $\xi_{s}$. One could try to work with just the quantities $\left|U_{c d}\right|^{2} \xi_{d}$ and $\left|U_{c s}\right|^{2} S \xi_{s}$ (which are what occur in the denominators in $R_{v}$ and $\left.R_{\bar{v}}\right)$. However, to extract these from the dimuon data one needs $B_{\mu}$, which depends on the charm hadronization and could be energy dependent. In particular, it is not accurately known near threshold. Also, the neutral-current cross sections depend separately on $S$, and finally there is a (very small) threshold effect in $\sigma^{\mathrm{NC}}$ from $c \rightarrow c$. It is therefore appropriate to employ a model for the charm threshold and to use the dimuon results as a consistency check.

Slow rescaling ${ }^{37}$ is a simple but reasonable prescription in which one calculates the $q \rightarrow c$ cross section in the parton model, but assigns the produced $c$ quark an effective mass $m_{c}$. The effect of this is that the usual quantities

$$
x q\left(x, Q^{2}\right)\left\{\begin{array}{c}
1 \\
(1-y)^{2}
\end{array}\right\},
$$

where $q$ is the appropriate quark or antiquark distribution, are replaced by

$$
z q\left(z, Q^{2}\right) \theta(1-z)\left\{\begin{array}{c}
1-y+x y / z \\
1-y+x\left(y^{2}-y\right) / z
\end{array}\right\},
$$

where $z \equiv x+m_{c}{ }^{2} / 2 M v$. [Some earlier analyses ${ }^{5,9}$ omitted the changes in the 1 and $(1-y)^{2}$ factors. That is not adequate for the present high-precision experiments.] For $m_{c}=1.5 \mathrm{GeV}$, slow rescaling predicts that charm production accounts for $7 \%$ of the $v N \rightarrow \mu^{-} X$ cross section, $\xi_{s} \simeq 0.65$, and $\xi_{d} \sim 0.85$ for typical CERN and Fermilab spectra. For $m_{c} \rightarrow 0$ (no suppression) or $m_{c} \rightarrow \infty$ (total suppression), $\sin ^{2} \theta_{W}$ changes drastically (by -0.014 and +0.034 , respectively), so it is essential to verify the slow rescaling prescription and to obtain a reasonable value of $m_{c}$.

There are a variety of conflicting theoretical estimates of $m_{c}$. The perturbative origin of $m_{c}$ would suggest a low (current-) quark mass of $\simeq 1.35 \mathrm{GeV}$. On the other hand, the $\theta(1-z)$ factor ensures that the hadronic invariant mass $W$ exceeds $\left(m_{c}{ }^{2}+m_{p}{ }^{2}\right)^{1 / 2}$. If one defines $m_{c}$ so that $W>m_{D}+m_{p}$ or $W>m_{\Lambda_{c}}$, then $m_{c} \simeq 2.6$ or $2.1 \mathrm{GeV}$, respectively. Of course, the definition $m_{c} \sim m_{J / \psi} / 2$ implies $1.55 \mathrm{GeV}$.

Since the appropriate definition of $m_{c}$ is unclear, it is best to regard it as a phenomenological parameter determined from the dimuon cross sections. The most precise results are from the CDHS Collaboration. ${ }^{127}$ They measured the ratios $R_{2}^{v} \equiv \sigma\left(\nu \rightarrow \mu^{-} \mu^{+}\right) / \sigma\left(\nu \rightarrow \mu^{-}\right)$and $R_{2}^{\bar{v}} \equiv \sigma\left(\bar{v} \rightarrow \mu^{+} \mu^{-}\right) / \sigma\left(\bar{v} \rightarrow \mu^{+}\right)$of dimuon to single muon cross sections as a function of $E_{v}\left(E_{\bar{v}}\right)$ and showed that slow rescaling with $m_{c}=1.5 \mathrm{GeV}$ correctly described the energy dependence of $R_{2}^{v}$ and $R_{2}^{\bar{v}}$ (after correcting for acceptances) above $\simeq 80 \mathrm{GeV}$. However, for $E<80 \mathrm{GeV}$ the measured dimuon cross sections are lower than the slow rescaling prediction (see Fig. 16 of Ref. 127). The main concern of CDHS was to extract $\left|U_{c d}\right|$ and $\left|U_{c s}\right|{ }^{2} S$ rather than to study the charm threshold, so they concentrated on the high-energy region.

For the neutral-current analysis, however, the lowenergy region is vital: around half of the events have $E_{v}<80 \mathrm{GeV}$ for a typical narrow band beam. If the suppression of $R_{2}^{v}$ for $E<80 \mathrm{GeV}$ were entirely due to a breakdown of slow rescaling, then the extracted $\sin ^{2} \theta_{W}$ would increase by $\sim 0.007$. Equivalently, the problem could be that a larger $m_{c}$ or a steeper $x s(x)$ distribution is required. On the other hand, an energy dependence of $B_{\mu}$ would affect $R_{2}^{v}$ but not the neutral-current analysis.

We have reconsidered the dimuon results using the structure functions described in Sec. III. Based on Mark III and other results ${ }^{129}$ we assume a larger $B_{\mu}$ than the value $(7.1 \pm 1.3 \%)$ used by CDHS: $B_{\mu}^{(a)} \sim 10 \%$ for $c^{(-)}$ produced by $\bar{d}, s$, or $\bar{s}$ and $B_{\mu}^{(b)}=7.5 \%$ for $c$ produced by $d$. This lower value for $d \rightarrow c$ is motivated by the low branching ratio $(\sim 4 \%)$ for $\Lambda_{c} \rightarrow \mu^{+}$and the assumption that $\Lambda_{c}$ is easier to produce in $d \rightarrow c$ than the other reactions.

The scale of $B_{\mu}$ does not affect the test of slow rescaling (which only depends on the energy dependence), but 


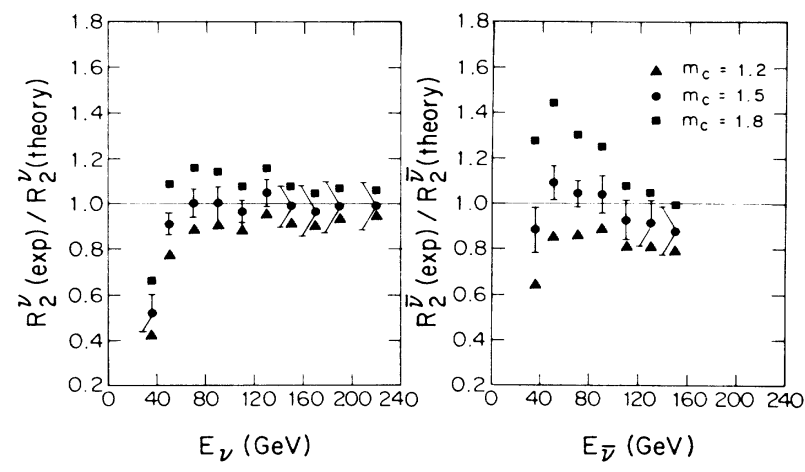

FIG. 12. (a) Ratio of measured $R_{2}^{v}=\sigma\left(\nu \rightarrow \mu^{-} \mu^{+}\right) /$ $\sigma\left(\nu \rightarrow \mu^{-}\right)$to the theoretical prediction from slow rescaling as a function of $E_{v}$. The theoretical calculation assumes $\eta_{H}=9.5$, $B_{\mu}^{a}=10 \%$, and $B_{\mu}^{b}=7.5 \%$, where $\eta_{H}$ is the $1-x$ power of $x s(x)$ at $Q_{0}{ }^{2}$ and $B_{\mu}^{a, b}$ are the muon branching ratios for ${ }_{c}^{(-)}$ produced from $(\bar{d}, s, \bar{s})$ or $d$, respectively. The ratios are shown for $m_{c}=1.2,1.5$, and $1.8 \mathrm{GeV}$. Error bars (which include only the experimental error in $R_{2}^{v}$ ) are shown for $m_{c}=1.5 \mathrm{GeV}$ only. (b) Same for $\bar{v}$.

it is important for the extraction of $U_{c d}$ for more than three fermion families. The possible energy dependence of $B_{\mu}$ is discussed below. The other major difference compared to the CDHS analysis is that (in accordance with later CDHS results ${ }^{130}$ ) we use a steeper $x$ distribution for $x s(x)$ :

$$
x s(x) \sim c(1-x)^{\eta_{H}},
$$

with $\eta_{H}=9.5$ at $5 \mathrm{GeV}^{2}$ (increasing to $\sim 11$ at $Q^{2} \sim 20$ $\mathrm{GeV}^{2}$ ) compared to $\eta_{H}=7$ used by CDHS. This provides an additional threshold suppression and removes some of the low- $E$ discrepancy.
The measured ratios of dimuon to single muon cross section divided by the slow rescaling prediction ${ }^{131}$ are shown in Fig. 12. Slow rescaling with $m_{c}=1.5 \pm 0.3$ $\mathrm{GeV}$ gives an excellent description of the data except for $E_{v} \leq 60 \mathrm{GeV}$. We suspect that the problem there is an energy dependence of $B_{\mu}$ rather than a breakdown of slow rescaling itself. This is motivated by the results of the Fermilab emulsion experiment ${ }^{132}$ E531, which finds that $\Lambda_{c}$ production in $v N$ is very important at low energies $(\simeq 30 \mathrm{GeV})$. This could easily give a lower $B_{\mu}$ near threshold and therefore resolve the discrepancy, but a definite conclusion will require better statistics. Supporting evidence is that the antineutrino data (for which $\bar{\Lambda}_{c}$ production should be much less important) shows much less structure at low $E_{\bar{v}}$.

The antineutrino data in Fig. 12(b) are consistent with slow rescaling but favor the lower values for $m_{c}$, while the CCFRR results ${ }^{128}$ (which have less statistics) favor the upper end of the $1.2-1.8-\mathrm{GeV}$ range.

Our conclusion is that slow rescaling with $m_{c}=1.5 \pm 0.3 \mathrm{GeV}$ gives a reasonable description of the dimuon data, with the $\pm 0.3 \mathrm{GeV}$ uncertainty implying an uncertainty in $\sin ^{2} \theta_{W}$ from deep-inelastic scattering of \pm 0.0041 . It should be emphasized that $m_{c}$ is an effective parameter that roughly incorporates the uncertainties from the slow rescaling formalism itself as well as those in the shapes of the $s$ (and to a smaller extent the $d$ and $\bar{d}$ ) distribution functions: An equivalent statement is that slow rescaling with $m_{c}=1.5 \mathrm{GeV}$ correctly describes dimuon (and therefore charm) production to within $15 \%$ when averaged over the appropriate spectra, implying an uncertainty of $<0.005$ in $\sin ^{2} \theta_{W}$. Finally, there is evidence for an energy dependence of $B_{\mu}$ near threshold, and the quantities in (A1) and (A2) extracted from the high-energy dimuon data are only weakly correlated with $m_{c}$.
*On leave from New York University, New York, NY 10003.

${ }^{1}$ S. Weinberg, Phys. Rev. Lett. 19, 1264 (1967); A. Salam, in Elementary Particle Theory, edited by N. Svartholm (Almquist and Wiksell, Stockholm, 1969), p. 367; S. L. Glashow, J. Iliopoulos, and L. Maiani, Phys. Rev. D 2, 1285 (1970).

${ }^{2}$ Previous global analyses include Abbott and Barnett (Ref. 3), Liede and Roos (Ref. 4), Kim, Langacker, Levine, and Williams (Ref. 5), Hung and Sakurai (Ref. 6), and Sehgal (Ref. 7). Klein and Schlenstedt (Ref. 8) have considered leptonic processes, Fogli (Ref. 9) has analyzed deep-inelastic neutrino scattering, and several authors (Ref. 10) have reviewed atomic parity violation. Klein (Ref. 11) has reviewed weakelectromagnetic interference, and Barbiellini and Santoni (Ref. 12) have reviewed weak-interaction experiments.

${ }^{3}$ L. F. Abbott and R. M. Barnett, Phys. Rev. D 18, 3214 (1978); 19, 3230 (1979).

${ }^{4}$ I. Liede and M. Roos, Phys. Lett. 82B, 89 (1979); Nucl. Phys. B167, 397 (1980).

${ }^{5}$ J. E. Kim, P. Langacker, M. Levine, and H. H. Williams, Rev. Mod. Phys. 53, 211 (1981).

${ }^{6}$ P. Q. Hung and J. J. Sakurai, Annu. Rev. Nucl. Part. Sci. 31, 375 (1981).

${ }^{7}$ L. M. Sehgal, Prog. Nucl. Part. Phys. 14, 1 (1985).
${ }^{8}$ M. Klein and S. Schlenstedt, Z. Phys. C 29, 235 (1985).

${ }^{9}$ G. L. Fogli, Nucl. Phys. B260, 593 (1985).

${ }^{10}$ E. D. Commins and P. H. Bucksbaum, Ann. Rev. Nucl. Part. Sci. 30, 1 (1980); C. A. Piketty, in Weak and Electromagnetic Interactions in Nuclei, edited by $\mathrm{H}$. V. Klapdor (Springer, Berlin, 1986), p. 603; E. N. Fortson and L. L. Lewis, Phys. Rep. 113, 289 (1984); M. A. Bouchiat and L. Pottier, Science 234, 1203 (1986).

${ }^{11}$ M. Klein, Fortschr. Phys. 33, 7 (1985).

${ }^{12} \mathrm{G}$. Barbiellini and C. Santoni, Riv. Nuovo Cimento 9, 1 (1986).

${ }^{13}$ We utilize the renormalization scheme of Sirlin (Ref. 14) in which $\sin ^{2} \theta_{W} \equiv 1-M_{W}{ }^{2} / M_{Z}{ }^{2}$. The radiative corrections calculated in the standard model are applied even in fits that allow the possibility of new physics on the grounds that the standard model is now clearly established as correct to first approximation.

${ }^{14}$ A. Sirlin, Phys. Rev. D 22, 971 (1980); 29, 89 (1984); W. J. Marciano and A. Sirlin, ibid. 22, 2695 (1980); 29, 75 (1984); 29, 945 (1984); Nucl. Phys. B189, 442 (1981); S. Sarantakos et al., ibid. B217, 84 (1983). Extensive references to other papers are given in Radiative Corrections in $S U_{2 L} \times U_{1}$, edited by B. W. Lynn and J. F. Wheater (World Scientific, 
Singapore, 1984). See also the recent papers of R. G. Stuart, Report No. CERN TH 4342-85 (unpublished), and F. Jegerlehner, Z. Phys. C 32, 195 (1986); 32, 435 (1986).

${ }^{15}$ Theoretical uncertainties are defined as uncertainties in the theoretical expressions and uncertainties due to parameters that are not measured in the same experiment.

${ }^{16}$ F. E. James and M. Roos, Comput. Phys. Commun. 10, 343 (1975).

${ }^{17} \mathrm{U}$. Amaldi et al. (in preparation).

${ }^{18}$ See, for example, B. W. Lynn and R. G. Stuart, Nucl. Phys. B253, 216 (1985), and references therein.

${ }^{19}$ H. Abramowicz et al., Phys. Rev. Lett. 57, 298 (1986).

${ }^{20}$ J. V. Allaby et al., Phys. Lett. 177B, 446 (1986).

${ }^{21}$ P. G. Reutens et al., Phys. Lett. 152B, 404 (1985); F. Merritt (private communication).

${ }^{22}$ D. Bogert et al., Phys. Rev. Lett. 55, 1969 (1985); F. Taylor (private communication).

${ }^{23} \mathrm{H}$. Abramowicz et al., Z. Phys. C 28, 51 (1985).

${ }^{24}$ V. V. Ammosov et al., Z. Phys. C 30, 569 (1986).

${ }^{25}$ P. A. Gorichev et al., Yad Fiz. 39, 626 (1984) [Sov. J. Nucl. Phys. 39, 396 (1984)].

${ }^{26}$ P. Bosetti et al., Nucl. Phys. B217, 1 (1983).

${ }^{27}$ D. Allasia et al., Phys. Lett. 133B, 129 (1983).

${ }^{28}$ J. Marriner et al., Phys. Rev. D 27, 2569 (1983).

${ }^{29}$ M. Jonker et al., Phys. Lett. 99B, 265 (1981); 103B, 469(E) (1981).

${ }^{30}$ F. S. Merritt et al., Phys. Rev. D 17, 2199 (1978).

${ }^{31}$ P. Wanderer et al., Phys. Rev. D 17, 1679 (1978).

${ }^{32}$ We do not include the 1977 CDHS results $R_{v}=0.293 \pm 0.010$, $R_{\bar{v}}=0.35 \pm 0.03$ [M. Holder et al., Phys. Lett. 71B, 222 (1977)] because of the larger systematic uncertainties compared with later CDHS experiments.

${ }^{33}$ C. H. Llewellyn Smith, Nucl. Phys. B228, 205 (1983).

${ }^{34}$ The CCFRR and FMM groups apply the more conventional QCD-improved parton-model analysis and obtain $0.242 \pm 0.012$ and $0.245 \pm 0.018$, respectively.

${ }^{35}$ P. Castorina and J. Mulders, Phys. Rev. D 31, 2753 (1985); 31, 2760 (1985); S. Fajfer and R. J. Oakes, ibid. 30, 1585 (1984).

${ }^{36}$ A. J. Buras and K. J. F. Gaemers, Nucl. Phys. B132, 249 (1978).

${ }^{37}$ R. M. Barnett, Phys. Rev. D 14, 70 (1976).

${ }^{38}$ We predict $r=0.455$ for CHARM, to be compared with their measured value $0.456 \pm 0.012$.

${ }^{39} \mathrm{We}$ are very grateful to F. Merritt (CCFRR) and F. Taylor (FMM) for providing this information.

${ }^{40}$ G. T. Jones et al., Phys. Lett. 178B, 329 (1986); R. W. L. Jones and S. Burke (private communication); S. J. Towers, Oxford thesis RAL T013, 1985.

${ }^{41}$ N. Armenise et al., Phys. Lett. 122B, 448 (1983); J. Moreels et al., ibid. 138B, 230 (1984).

${ }^{42}$ T. Kafka et al., Phys. Rev. Lett. 48, 910 (1982).

${ }^{43}$ D. D. Carmony et al., Phys. Rev. D 26, 2965 (1982).

${ }^{44}$ J. Blietschau et al., Phys. Lett. 88B, 381 (1979).

${ }^{45}$ F. A. Harris et al., Phys. Rev. Lett. 39, 437 (1977).

${ }^{46}$ Gorichev et al. (Ref. 25).

${ }^{47}$ Marriner et al. (Ref. 28).

${ }^{48}$ Allasia et al. (Ref. 27).

${ }^{49}$ See, for example, CDHS Collaboration, H. Abramowicz et al., Z. Phys. C 25, 29 (1984); WA25 BEBC Collaboration, D. Allasia et al., Phys. Lett. 135B, 231 (1984); for a review, see F. Sciulli, in Proceedings of the Twelfth International Symposium on Lepton and Photon Interactions at High Energies, Kyoto, Japan, 1985, edited by M. Konuma and K.
Takahashi (Research Institute for Fundamental Physics, Kyoto University, Kyoto, 1986), p. 8.

${ }^{50} \mathrm{~K}$. Abe et al., Phys. Rev. Lett. 56, 1107 (1986); 56, 1183(E) (1986); L. A. Ahrens et al., Phys. Rev. D 35, 785 (1987).

${ }^{51}$ H. J. Grabosch et al., Z. Phys. C 31, 203 (1986).

${ }^{52}$ C. Baltay et al., Phys. Rev. Lett. 57, 2629 (1986).

${ }^{53}$ F. Bergsma et al., Phys. Lett. 157B, 469 (1985).

${ }^{54} \mathrm{H}$. Faissner et al., Phys. Lett. 125B, 230 (1983).

${ }^{55} \mathrm{We}$ include only the recent BNL E734 experiment because of its smaller systematic uncertainties. For earlier experiments see Ref. 56, and references therein.

${ }^{56}$ J. Horskotte et al., Phys. Rev. D 25, 2743 (1982).

${ }^{57}$ T. Kitigaki et al., Phys. Rev. D 28, 436 (1983), and references therein.

${ }^{58} \epsilon=0.125 \pm 0.125$ is suggested both by theory (Ref. 5) and the E734 data. The uncertainty from $\epsilon$ is strongly correlated to $M_{A}$ and is therefore not considered separately.

${ }^{59}$ See, D. Rein and L. M. Sehgal, Nucl. Phys. B223, 29 (1983).

${ }^{60}$ For a review, see G. L. Fogli, Phys. Rev. D 34, 751 (1986); Nucl. Phys. B207, 322 (1982).

${ }^{61}$ E. Pasierb et al., Phys. Rev. Lett. 43, 96 (1979).

${ }^{62}$ L. A. Ahrens et al., Phys. Rev. Lett. 51, 1514 (1983); 54, 18 (1985).

${ }^{63}$ F. Bergsma et al., Phys. Lett. 117B, 272 (1982), 147B, 481 (1984); C. Santoni (private communication).

${ }^{64}$ A. M. Cnops et al., Phys. Rev. Lett. 41, 357 (1978); M. F. Bregman, thesis, Nevis Report No. 248, 1984; M. Murtagh (private communication).

${ }^{65}$ R. H. Heisterberg et al., Phys. Rev. Lett. 44, 635 (1980).

${ }^{66} \mathrm{~N}$. Armenise et al., Phys. Lett. 86B, 225 (1979).

${ }^{67}$ H. Faissner et al., Phys. Rev. Lett. 41, 213 (1978).

${ }^{68} \mathrm{~J}$. Blietschau et al., Nucl. Phys. B114, 189 (1976).

${ }^{69}$ F. Reines et al., Phys. Rev. Lett. 37, 315 (1976).

${ }^{70}$ R. C. Allen et al., Phys. Rev. Lett. 55, 2401 (1985).

${ }^{71} \mathrm{We}$ have increased the systematic uncertainty in the VMWOF experiment (Ref. 65) from 0.2 to 0.4 to account for uncertainties in the background subtraction, efficiency, and normalization.

${ }^{72}$ P. Vogel et al., Phys. Rev. C 24, 1543 (1981); K. Schreckenbach et al., Phys. Lett. 160B, 325 (1985).

${ }^{73} \mathrm{We}$ are very grateful to $\mathrm{P}$. Vogel for advice on reactor spectra.

${ }^{74}$ M. A. Bouchiat et al., Phys. Lett. 117B, 358 (1982); 134B, 463 (1984); CNRS report (unpublished).

${ }^{75}$ S. L. Gilbert et al., Phys. Rev. Lett. 55, 2680 (1985); Phys. Rev. A 34, 792 (1986).

${ }^{76}$ P. S. Drell and E. D. Commins, Phys. Rev. Lett. 53, 968 (1984).

${ }^{77}$ T. P. Emmons et al., Phys. Rev. Lett. 51, 2089 (1983).

${ }^{78}$ H. Hollister et al., Phys. Rev. Lett. 46, 643 (1981).

${ }^{79}$ G. N. Birich et al., Zh. Eksp. Teor. Fiz. 87, 776 (1984) [Sov. Phys. JETP 60, 442 (1985)].

${ }^{80}$ J. D. Taylor, Oxford thesis; P. G. H. Sandars (private communication).

${ }^{81}$ We have omitted the early null experiments (Ref. 10), the Novosibirsk bismuth $(648 \mathrm{~nm})$ experiment $[-20.2 \pm 2.7, \mathrm{~L}$. M. Barkov and M. S. Zolotorev, Phys. Lett. 85B, 308 (1979)], which is clearly inconsistent with the Moscow and Oxford results, and the original Berkeley thallium result $\left[-1.80_{-0.60}^{+0.65}\right.$, P. H. Bucksbaum et al., Phys. Rev. D 24, 1134 (1981)].

${ }^{82}$ M. A. Bouchiat et al., CNRS report (unpublished); and private communication.

${ }^{83}$ C. Y. Prescott et al., Phys. Lett. 77B, 347 (1978); 84B, 524 
(1979).

${ }^{84}$ A. Argento et al., Phys. Lett. 120B, 245 (1983); 140B, 142 (1984).

${ }^{85}$ UA2 Collaboration, R. Ansari et al., Phys. Lett. 186B, 440 (1987).

${ }^{86}$ UA1 Collaboration, G. Arnison et al., Phys. Lett. 166B, 484 (1986).

${ }^{87}$ H. J. Behrend et al., Z. Phys. C 14, 283 (1982); Report No. DESY-87-02 (unpublished).

${ }^{88}$ W. Bartel et al., Z. Phys. C 30, 371 (1986); B. Naroska, Phys. Rep. 148, 67 (1987)

${ }^{89}$ B. Adeva et al., Phys. Rev. Lett. 55, 665 (1985); J. Mnich, thesis, RWTH, Aachen, 1987.

${ }^{90} \mathrm{Ch}$. Berger et al., Z. Phys. C 27, 341 (1985).

${ }^{91}$ R. Brandelik et al., Phys. Lett. 110B, 173 (1982); H.-U. Martyn, in Proceedings of the XXII Rencontre de Moriond, Les Arcs, 1987 (unpublished).

${ }^{92}$ D. Bender et al., Phys. Rev. D 30, 515 (1984); M. Derrick et al., ibid. 31, 2352 (1985).

${ }^{93}$ M. E. Levi et al., Phys. Rev. Lett. 51, 1941 (1983).

${ }^{94}$ W. W. Ash et al., Phys. Rev. Lett. 55, 1831 (1985).

${ }^{95} \mathrm{Ch}$. Berger et al., Z. Phys. C 21, 53 (1983); H. Kapitza, Hamburg thesis, 1984.

${ }^{96}$ M. Althoff et al., Z. Phys. C 22, 13 (1984); 26, 521 (1985).

${ }^{97}$ H. J. Behrend et al., Phys. Lett. 114B, 282 (1982); CELLO Collaboration, contributed paper, XXIII International Conference on High Energy Physics, Berkeley, California, 1986 (unpublished).

${ }^{98}$ W. Bartel et al., Phys. Lett. 161B, 188 (1985); Naroska (Ref. 88 ).

${ }^{99}$ B. Adeva et al., Phys. Lett. 179B, 177 (1986).

${ }^{100} \mathrm{Ch}$. Berger et al., Z. Phys. C 28, 1 (1985).

${ }^{101}$ K. K. Gan et al., Phys. Lett. 153B, 116 (1985).

${ }^{102}$ H. J. Behrend et al., Phys. Lett. 114B, 282 (1982); Report No. DESY-87-02 (unpublished).

${ }^{103}$ E. Fernandez et al., Phys. Rev. Lett. 54, 1620 (1985).

${ }^{104}$ The theoretical uncertainties should be interpreted as being equivalent to $1 \sigma$ errors.

${ }^{105}$ The effects of nondegenerate heavy-fermion doublets are absorbed in the radiative corrections parameters $\rho^{\mathrm{NC}}$ and $\kappa$ defined in Sec. II.

${ }^{106} \mathrm{~A}$ fit to the most precise experiments of each type yields $\rho=0.998 \pm 0.0089$ and $\sin ^{2} \theta_{W}=0.229 \pm 0.0066$. The first six reactions in Table XII plus $e^{+} e^{-}$and coherent pion data yield $\rho=0.996 \pm 0.0084, \sin ^{2} \theta_{W}=0.227 \pm 0.0064$.

${ }^{107}$ For two parameters an allowed region at $90 \%$ C.L. corresponds to an increase $\Delta \chi^{2}(0.90,2)=4.6$ above the minimum $\chi^{2}$ value, while the $68 \%$-C.L. region is defined by $\Delta \chi^{2}(0.68,2)=2.3$. For a single parameter the corresponding values are $\Delta \chi^{2}(0.90,1)=2.7$ and $\Delta \chi^{2}(0.68,1)=1.0$. In all cases, the $\chi^{2}$ value at each point has been minimized with respect to all other free parameters. In a few cases physical constraints (such as regions bounding on infinity or the positivity of masses) require a modification of these $\Delta \chi^{2}$ values, with $90 \%$-C.L. regions typically coinciding with naive $80 \%$ C.L. contours.

${ }^{108}$ The $90 \%$-C.L. limits on $m_{t}$ should not be confused with the projection of the $90 \%$-C.L. contours in Fig. 5 on the $m_{t}$ axis. The former refers to the probability distribution in $m_{t}$ alone, defined as $P\left(m_{t}\right)=\int_{0}^{1} P\left(m_{t}, \sin ^{2} \theta_{W}\right) d \sin ^{2} \theta_{W}$.

${ }^{109} \mathrm{~A}$ heavy fourth family could also in principle relax the constraints on the Kobayashi-Maskawa-Cabibbo angles if its mixings with the first two families is large. This would increase the $\sin ^{2} \theta_{W}$ from deep-inelastic scattering and would make the limits on the mass splittings more stringent.

${ }^{110}$ M. B. Einhorn, D. R. T. Jones, and M. Veltman, Nucl. Phys. B191, 146 (1981); S. Bertolini, ibid. B272, 77 (1986); J. J. van der Bij and M. Veltman, ibid. B231, 205 (1984); J. J. van der Bij, ibid. B248, 141 (1984).

${ }^{111}$ This constraint is weaker than in previous analyses because of our use of a new spectrum and inclusion of theoretical spectrum uncertainties.

${ }^{112}$ If one assumes $h_{A A}=g_{A}^{e 2}$ and $h_{V V}=g_{V}^{e 2}$ then the $e^{+} e^{-}$data are consistent with solutions $\mathrm{A}$ and $\mathrm{C}$ and exclude the vector-dominant solution (B). This assumption holds to the extent that the neutral current is dominated by the exchange of a single $Z$ boson. It is therefore reasonable to assume that the ve couplings of the $Z$ are uniquely determined. However, the $h_{i i}=g_{i}^{e 2}$ assumption is violated by most extensions of the standard model, so we do not include the $e^{+} e^{-}$data in the $g_{V}^{e}-g_{A}^{e}$ fits.

${ }^{113}$ The allowed region is similar to Fig. 24 of Ref. 5.

${ }^{114}$ There have been several recent analyses (Refs. 115 and 116) of extra $Z$ bosons based on earlier data sets.

${ }^{115}$ L. S. Durkin and P. Langacker, Phys. Lett. 166B, 436 (1986).

${ }^{116}$ D. London and J. Rosner, Phys. Rev. D 34, 1530 (1986); E. Cohen et al., Phys. Lett. 165B, 76 (1985); J. Ellis et al., Nucl. Phys. B276, 14 (1986); V. Barger, N. G. Deshpande, and K. Whisnant, Phys. Rev. Lett. 56, 30 (1986); Phys. Rev. D 35, 1005 (1987); J. Rosner, Commun. Nucl. Part. Phys. 14, 229 (1985); F. del Aguila et al., Nucl. Phys. B283, 50 (1987); B284, 530 (1987); P. J. Franzini and F. Gilman, Phys. Rev. D 35, 855 (1987); V. Barger et al., ibid. 35, 2893 (1987).

${ }^{117}$ For a review, see P. Langacker, in Proceedings of the Twelfth International Symposium on Lepton and Photon Interactions at High Energy (Ref. 49), p. 186.

${ }^{118}$ See Ref. 115 for more details and a complete set of references.

${ }^{119}$ See, for example, M. J. Duncan and P. Langacker, Nucl. Phys. B277, 285 (1986).

${ }^{120}$ In some models comparable limits have been obtained from electroweak radiative corrections to charged-current data: W. J. Marciano and A. Sirlin, Phys. Rev. D 35, 1672 (1987).

${ }^{121}$ W. A. Bardeen, A. Buras, D. Duke, and T. Muta, Phys. Rev. D 18, 3998 (1978).

${ }^{122} \mathrm{~W}$. Marciano and A. Sirlin, in Proceedings of the Second Workshop on Grand Unification, Ann Arbor, Michigan, 1981, edited by J. Leveille, L. Sulak, and D. Unger (Birkhauser, Boston, 1981).

${ }^{123}$ The uncertainty quoted for $\sin ^{2} \hat{\theta}_{W}\left(M_{W}\right)$ is smaller than the $\sin ^{2} \theta_{W}$ uncertainty (0.0048) in Sec. IV A because the latter includes a nontrivial (0.002) component from $m_{t}$ and $M_{H}$, while in this section we keep $m_{t}$ and $M_{H}$ fixed. As in apparent from Table XVI, $\sin ^{2} \hat{\theta}_{W}\left(M_{W}\right)$ is less sensitive to $m_{t}$ than is $\sin ^{2} \theta_{W}$.

${ }^{124} \mathrm{H}$. Georgi and S. Glashow, Phys. Rev. Lett. 32, 438 (1974); H. Georgi, H. Quinn, and S. Weinberg, ibid. 33, 451 (1974).

${ }^{125}$ D. S. Ayres et al., in Proceedings of the 1986 Summer Study, Snowmass, Colorado, 1986 (unpublished).

${ }^{126}$ S. Dimopoulos, S. Raby, and F. Wilczek, Phys. Rev. D 24, 1681 (1981); L. Ibanez and G. Ross, Phys. Lett. 105B, 439 (1981); M. B. Einhorn and D. R. T. Jones, Nucl. Phys. B196, 475 (1982); W. Marciano and G. Senjanovic, Phys. Rev. D 25, 3092 (1982).

${ }^{127}$ CDHS Collaboration, H. Abramowicz et al., Z. Phys. C 15, 19 (1982).

${ }^{128}$ CCFRR Collaboration, K. Lang, Rochester thesis, UR-908, 
1985.

${ }^{129}$ R. M. Baltrusaitis et al., Phys. Rev. Lett. 54, 1976 (1985); see also Lang (Ref. 128), and Sciulli (Ref. 49).

${ }^{130}$ CDHS group (private communication).
${ }^{131}$ It is not entirely a prediction since the value of $S$ was extracted from the high-energy dimuon results.

${ }^{132}$ N. Ushida et al., Phys. Lett. 121B, 287 (1983). 